\title{
Comprehensive behavioral analysis of mice deficient in Rapgef2 and Rapgef6, a subfamily of guanine nucleotide exchange factors for Rap small GTPases possessing the Ras/Rap-associating domain
}

\author{
Kazuhiro Maeta ${ }^{1,3+}$, Satoko Hattori ${ }^{2+}$, Junji Ikutomo ${ }^{1}$, Hironori Edamatsu', Shymaa E. Bilasy ${ }^{1,4}$,
}

Tsuyoshi Miyakawa ${ }^{2}$ and Tohru Kataoka ${ }^{1 *}$

\begin{abstract}
Rapgef2 and Rapgef6 define a subfamily of guanine nucleotide exchange factors for Rap small GTPases, characterized by the possession of the Ras/Rap-associating domain. Previous genomic analyses suggested their possible involvement in the etiology of schizophrenia. We recently demonstrated the development of an ectopic cortical mass (ECM), which resembles the human subcortical band heterotopia, in the dorsal telencephalon-specific Rapgef2 conditional knockout (Rapgef2-cKO) brains. Additional knockout of Rapgef6 in Rapgef2-cKO mice resulted in gross enlargement of the ECM whereas knockout of Rapgef6 alone (Rapgef6-KO) had no discernible effect on the brain morphology. Here, we performed a battery of behavioral tests to examine the effects of Rapgef2 or Rapgef6 deficiency on higher brain functions. Rapgef2-cKO mice exhibited hyperlocomotion phenotypes. They showed decreased anxiety-like behavior in the elevated plus maze and the open-field tests as well as increased depression-like behavior in the Porsolt forced swim and tail suspension tests. They also exhibited increased sociability especially in novel environments. They showed defects in cognitive function as evidenced by reduced learning ability in the Barnes circular maze test and by impaired working memory in the T maze tests. In contrast, although Rapgef6 and Rapgef2 share similarities in biochemical roles, Rapgef6-KO mice exhibited mild behavioral abnormalities detected with a number of behavioral tests, such as hyperlocomotion phenotype in the open-field test and the social interaction test with a novel environment and working-memory defects in the T-maze test. In conclusion, although there were differences in their brain morphology and the magnitude of the behavioral abnormalities, Rapgef2-cKO mice and Rapgef6-KO mice exhibited hyperlocomotion phenotype and working-memory defect, both of which could be recognized as schizophrenia-like behavior.
\end{abstract}

Keywords: Rap small GTPases, Guanine nucleotide exchange factors, Rapgef2, Rapgef6, Behavioral analysis, Schizophrenia

\footnotetext{
*Correspondence: kataoka@people.kobe-u.ac.jp

tEqual contributors

${ }^{1}$ Division of Molecular Biology, Department of Biochemistry and Molecular Biology, Kobe University Graduate School of Medicine, 7-5-1 Kusunoki-cho, Chuo-ku, Kobe 650-0017, Japan

Full list of author information is available at the end of the article
}

(c) The Author(s). 2018 Open Access This article is distributed under the terms of the Creative Commons Attribution 4.0 International License (http://creativecommons.org/licenses/by/4.0/), which permits unrestricted use, distribution, and reproduction in any medium, provided you give appropriate credit to the original author(s) and the source, provide a link to the Creative Commons license, and indicate if changes were made. The Creative Commons Public Domain Dedication waiver (http://creativecommons.org/publicdomain/zero/1.0/) applies to the data made available in this article, unless otherwise stated. 


\section{Introduction}

Rap proteins (Rap1A, Rap1B, Rap2A, Rap2B and Rap2C) belong to the Ras-family of small GTPases and are implicated in the regulation of a variety of cellular phenomena including proliferation, adhesion, polarity and endocytosis [1]. In the nervous system, Rap proteins have been demonstrated to play important roles in neural development during embryogenesis as well as in synaptic remodeling and plasticity in differentiated neurons [2-8]. During embryogenesis, Rap1 function as master regulators of neural cell polarity in the neocortical development and their loss leads to severe brain malformations in mice [5]. In differentiated neurons, Rap1 and Rap2 are involved in long-term depression, cortico-amygdala plasticity, fear learning, spatial learning and fear extinction [6, 9-12]. Guanine nucleotide exchange factors (GEFs) function as activators of small GTPases by catalyzing the release of GDP from the inactive GDP-bound forms, thereby accelerating GTP loading to yield the active GTP-bound forms [13]. To date, more than 10 GEFs specific for Rap have been reported. They are regulated by distinct mechanisms and responsible for differential regulation of Rap1 activity in spatial, temporal and celltype-specific manners [1]. A number of Rap1-GEFs are known to play important roles in development and function in the nervous system. For example, Rapgef4, also named Epac2 and cAMP-GEF1, activated by cAMP binding promotes spine shrinkage, resulting in synapse structural destabilization in cultured rat cortical neurons [14]. Moreover, a mutation in the Rapgef4 gene was associated with autism in humans, and the overexpression of this Rapgef4 mutant resulted in an impairment of basal dendrite maintenance in mice [15]. Combined deletion of Rapgef4 and its paralogue Rapgef3, also named Epac1 and cAMP-GEF1, in the forebrain caused defects in spatial learning and social interaction in mice [16]. Rapgef1, also named C3G, is responsible for Rap1 activation downstream of the Reelin signaling and plays a crucial role in neural development, in particular neuronal migration [17].

Rapgef2, also named RA-GEF-1, PDZ-GEF1, CNrasGEF and nRapGEP [18-21], and Rapgef6, also named RA-GEF-2 and PDZ-GEF2 [22], constitute a unique Rap1-GEF subfamily characterized by the possession of the Ras/Rap-associating (RA) domains capable of associating with the GTP-bound forms of Rap1 and M-Ras and the PSD-95/DlgA/ZO-1 (PDZ) domains. We previously showed that dorsal telencephalon-specific conditional Rapgef2 knockout (Rapgef2-cKO) mice developed severe brain malformations including an ectopic cortical mass (ECM) extending throughout the rostro-caudal axis of the cerebral hemisphere, enlargement of the lateral ventricles, interruption of the pyramidal cells in the hippocampal CA1 region and agenesis of interhemispheric connections [23-25]. We also reported that Rapgef2-cKO mice exhibited a lower seizure threshold to pilocarpine-induced status epilepticus [24]. Further analyses of their brain development revealed that they exhibit severe defects in formation of apical surface adherence junctions and in the development of radial glial cells (RGCs) [26]. Additional knockout of Rapgef6 in Rapgef2-cKO mice (Rapgef2/6-dKO) resulted in marked enlargement of the ECM and aggravation of the RGC developmental defects, suggesting that Rapgef6 shares neural functions with Rapgef2 [26]. On the other hand, knockout of Rapgef6 alone (Rapgef6-KO) had no discernible effect on the brain morphology [26, 27]. Moreover, human genetic studies in a cohort of schizophrenia patients showed strong genetic association of rare inherited copy number variations involving RAPGEF2 and RAPGEF6 with schizophrenia $[28,29]$. Moreover, the ECM formed in Rapgef2-cKO mice resembles that found in human subcortical band heterotopia patients, who display symptoms of mental retardation and epilepsy [30]. In Rapgef2-cKO mice, the malformation of the commissural system and agenesis of the corpus callosum (CC) [24], were also reported to be associated with schizophrenia-like behavior in mice carrying mutations in the Disrupted in Schizophrenia-1 (DISC-1) gene [31-34]. These results prompted us to perform comprehensive behavioral analysis in order to gain insights into the role of these Rap activators in the regulation of higher brain functions.

\section{Results}

\section{General health and general behavioral characteristics}

Rapgef2-cKO mice, Rapgef2 was specifically disrupted in the dorsal telencephalon, were created by mating Rapgef $2^{\text {flox/flox }}$ mice with $E m x 1^{\text {cre/+ }}$ mice expressing Cre recombinase under the control of the $E m x 1$ promoter [24]. To assess the effects of Rapgef2 deficiency, we compared Rapgef2-cKO mice and Rapgef $2^{\text {flox/flox }} ; E m \times 1^{+/+}$ mice in this study. We also assessed the effects of Rapgef6 deficiency by comparing Rapgef6-KOmice and Rapgef6 ${ }^{+/+}$ (wild-type) mice [35]. We performed the experiments using Rapgef2-cKO mice and Rapgef $2^{\text {flox/flox }} ; E m \times 1^{+/+}$mice (herein after referred to as control mice), and then performed those using Rapgef6-KO mice and wild-type mice. In our experimental model, it was difficult to compare between control mice and wild-type mice because of the differences in the factors affecting the results, such as the number of backcrossing and environmental factors. In Rapgef2-cKO mice, the body weight and temperature were comparable to those in the control mice (Fig. 1a, $\mathrm{F}_{1,43}=1.745, p=0.1935$; Fig. $1 \mathrm{~b}, \mathrm{~F}_{1,43}=$ 1.991, $p=0.1654)$. Although the grip strength was not significantly affected by Rapgef2 genotype (Fig. 1c, $\left.\mathrm{F}_{1,43}=1.149, p=0.2897\right)$, the wire-hang test demonstrated a significant decrease in the latency to fall in Rapgef2-cKO mice (Fig. 1d, $\mathrm{F}_{1,43}=16.785, p=0.0002$ ). In Rapgef6-KO mice, the bodyweight was significantly 


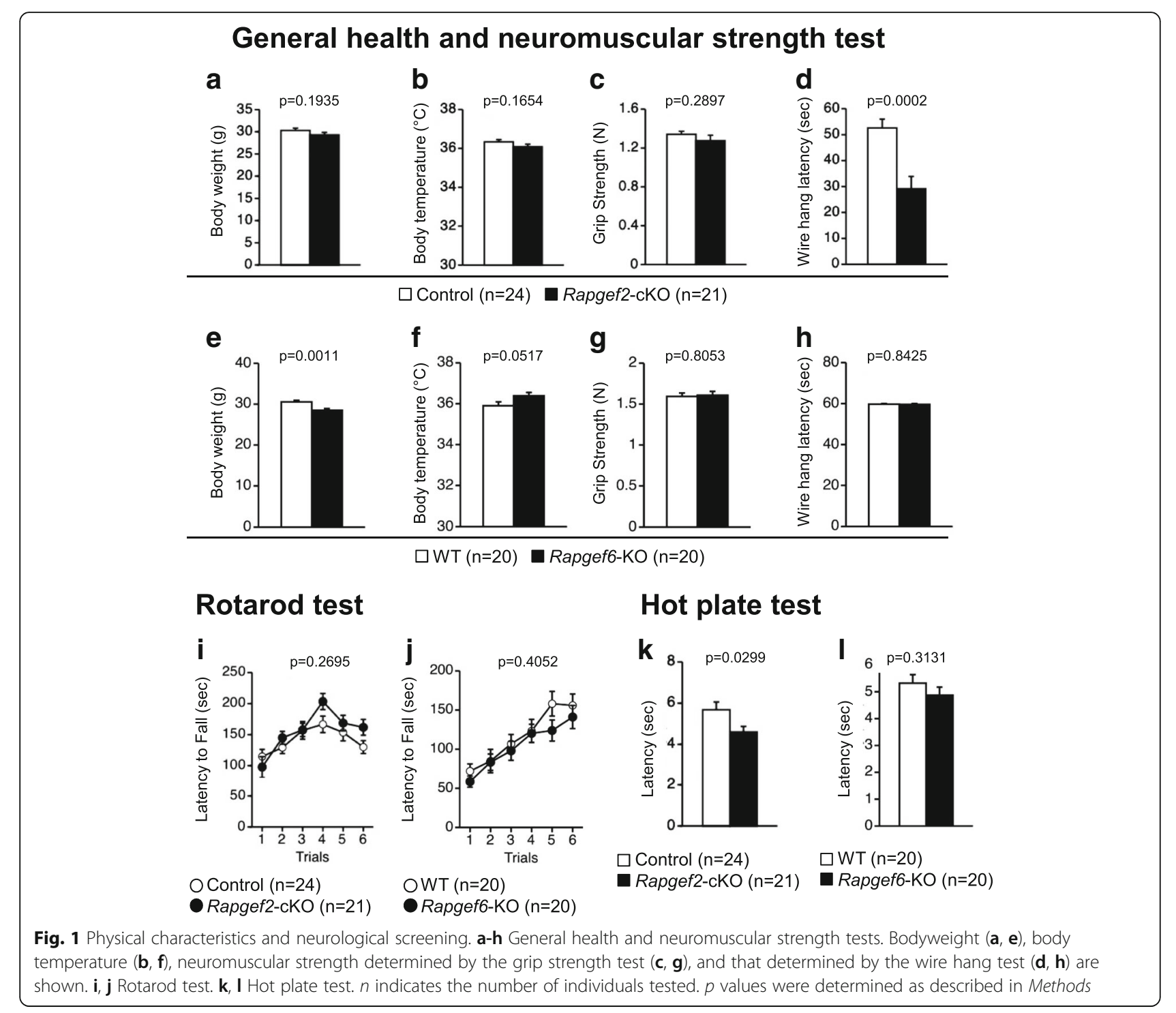

lighter (Fig. 1e, $F_{1,38}=12.541, p=0.0011$ ) and the body temperature was relatively higher than the wild-type littermates (Fig. 1f, $\mathrm{F}_{1,38}=4.036, p=0.0517$ ). Rapgef6 genotype did not seem to affect the grip strength and the latency to fall in the wire-hang test (Fig. $1 \mathrm{~g}, \mathrm{~F}_{1,38}=0.062, p=0.8053$; Fig. $1 \mathrm{~h}, \mathrm{~F}_{1,38}=0.04, p=0.8425$, respectively). In the accelerating rotarod test, although the differences associated with the Rapgef2 or Rapgef6 genotype were not statistically significant, we observed a slight tendency for increased latency to fall in Rapgef2-cKO mice (Fig. 1i, genotype effect, $\mathrm{F}_{1,43}=1.251, p=0.2695$; trial effect, $\mathrm{F}_{5,215}=14.382$, $p<0.0001$; genotype $\times$ trial interaction, $\mathrm{F}_{1,43}=2.051$, $p=0.0728 ;$ 4th trial, genotype effect, $\mathrm{F}_{1,43}=3.86, p=0$. 0559; 5th trial, genotype effect, $\mathrm{F}_{1,43}=0.649, p=0.4249$; 6th trial, genotype effect, $\mathrm{F}_{1,43}=3.852, p=0.0562$; Fig. $1 \mathrm{j}$, genotype effect, $\mathrm{F}_{1,38}=0.708, p=0.4052$; trial effect, $\mathrm{F}_{5,190}=34$. $155, p<0.0001$; genotype $\times$ trial interaction, $\mathrm{F}_{5,190}=1.127$, $p=0.3477)$. In the hot plate pain test, the latency to the first hind-paw response was significantly decreased in Rapgef2cKO mice (Fig. 1k, $\mathrm{F}_{1,43}=5.043, p=0.0299$ ) but not in Rapgef6-KO mice (Fig. 11, $\mathrm{F}_{1,38}=1.045, p=0.3131$ ).

\section{Effects on locomotor activity and anxiety-like behavior}

We performed the open field test with a novel environment to examine the effects on the locomotor activity. In all of the time windows examined, Rapgef2-cKO mice traveled longer in distance than control mice (Fig. 2a, genotype effect, $\mathrm{F}_{1,43}=36.348, p<0.0001$; time effect; $\mathrm{F}_{23,989}=70.365, p<0.0001$; genotype $\times$ time interaction, $\left.\mathrm{F}_{23,989}=5.174, p<0.0001\right)$. In contrast, Rapgef6- $\mathrm{KO}$ mice did not clearly exhibit difference in the total distance traveled (Fig. 2c, genotype effect, $\mathrm{F}_{1,38}=0.03, p=0.8638$ ). However, the time-dependent changes appeared to be affected by the Rapgef6 genotype (Fig. 2c, time effect, $\mathrm{F}_{23,874}=84.27, p<0.0001$; genotype $\times$ time interaction, $\left.\mathrm{F}_{23,874}=2.350, p=0.0004\right)$. These results suggest that 


\section{Open field test}

a

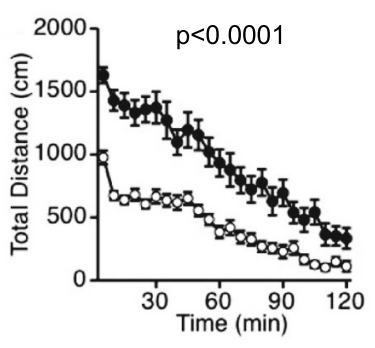

OControl $(n=24)$ b

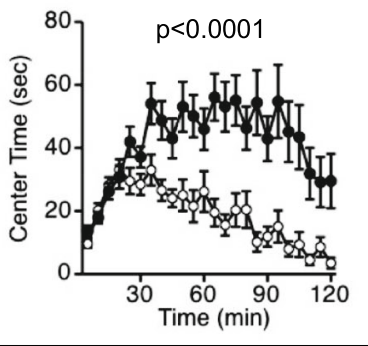

Rapgef2-cKO $(\mathrm{n}=21)$
C

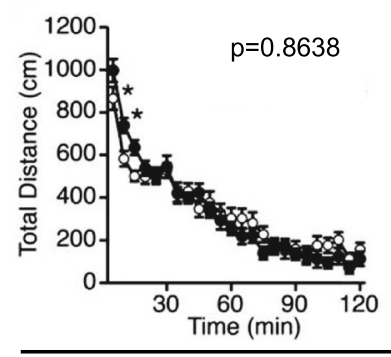

d

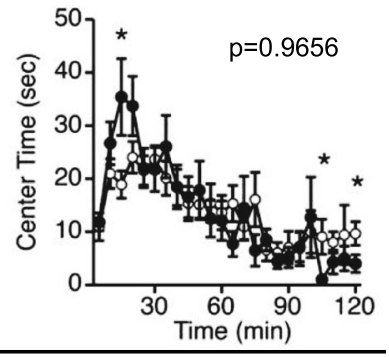

OWT $(n=20)$ Rapgef6-KO $(n=20)$

\section{Light/dark transition test}

e

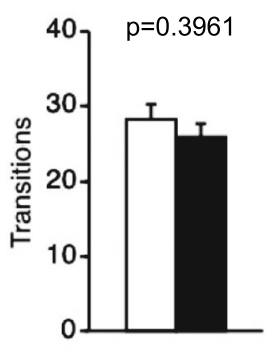

$\square$ Control $(n=24)$ f

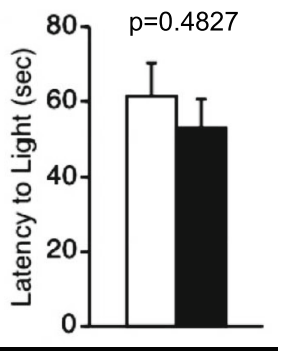

Rapgef2-cKO $(\mathrm{n}=21)$ g

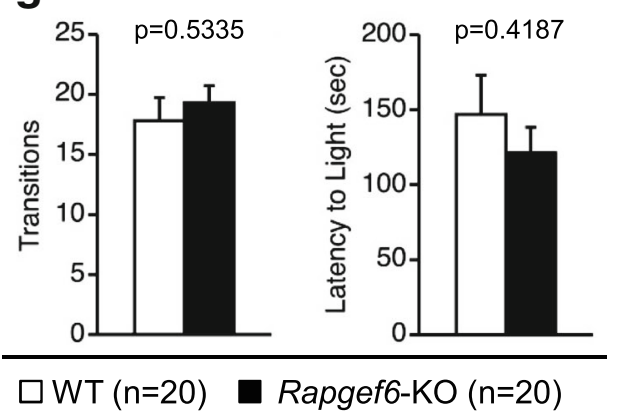

\section{Elevated plus maze test}
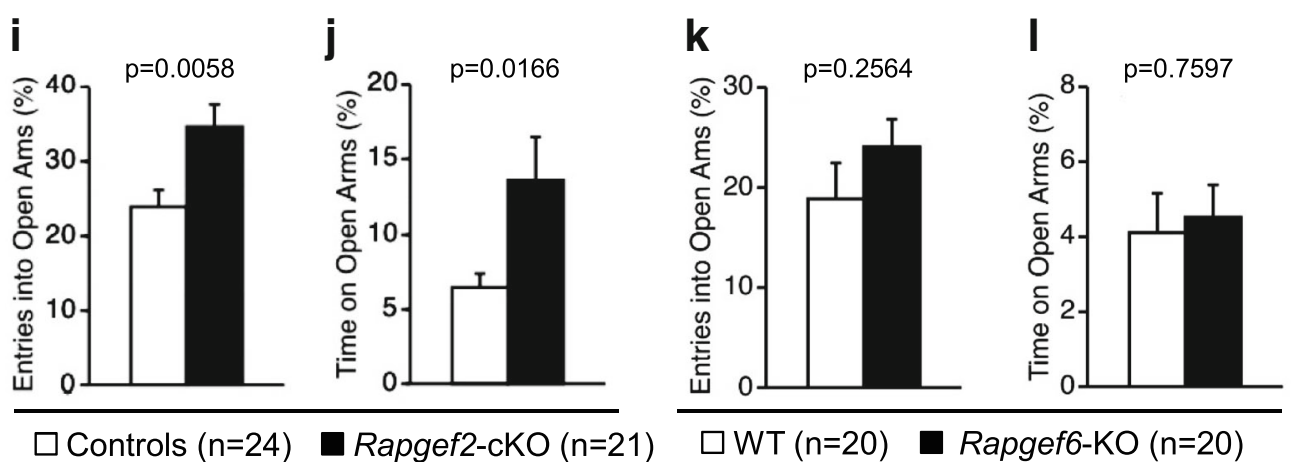

Fig. 2 Assessment for locomotor activity and anxiety-like behavior. a-d Open-field test in a novel environment. Total distance traveled (a, c) and time spent in arena center $(\mathbf{b}, \mathbf{d})$ were determined. $\mathbf{e}-\mathbf{h}$ Light/dark transition test. The number of light/dark transitions (e, $\mathbf{g})$ and the first latency to enter the light chamber $(\mathbf{f}, \mathbf{h})$ were determined. $\mathbf{i}-\mathbf{I}$ Elevated plus maze test. Percentages of entries into the open arms (i, $\mathbf{k})$ and those of time spent in the open arms (j, I) were determined. $n$ indicates the number of individuals tested. $p$ values were determined as described in $M e t h o d s .{ }^{*}, p<0.05$

Rapgef2-cKO mice strongly exhibited hyperlocomotion phenotypes whereas Rapgef6-KO mice weakly exhibited hyperlocomotion phenotypes consistent with previous observations [27].

In the open field test, the time spent exploring the center area is generally considered as an index of anxiety. The time spent in the arena center was significantly increased in Rapgef2-cKO mice (Fig. 2b, $\mathrm{F}_{1,43}=19.658$, $p<0.0001)$. The time spent in the arena center was also affected by the Rapgef6 genotype in a time-dependent manner (Fig. 2d, genotype effect, $\mathrm{F}_{1,38}=0.002, p=0.9656$; genotype $\times$ time interaction, $\mathrm{F}_{23,874}=1.618, p=0.0334$; time effect, $\left.\mathrm{F}_{23,874}=10.426, p<0.0001\right)$. Rapgef6-KO mice spent more time in the arena center in the initial phase of the test although they stayed in the peripheral arena in later phases (Fig. 2d).

To further assess the impacts on anxiety-like behavior, we performed a light/dark transition and elevated plus 
maze tests. The light/dark transition test failed to detect the changes associated with the genotypes of Rapgef 2 or Rapgef6; the number of transitions between the light and dark chambers was not significantly different among the examined groups (Rapgef2-cKO, Fig. 2e, $\mathrm{F}_{1,43}=0.735$, $p=0.3961$; Rapgef6-KO, Fig. 2g, $\mathrm{F}_{1,38}=0.395, p=0.5335$ ), the latency to first enter the light chamber (Rapgef2-cKO, Fig. 2f, $\mathrm{F}_{1,43}=0.501, p=0.4827$; Rapgef6-KO, Fig. 2h, $\left.\mathrm{F}_{1,38}=0.669, p=0.4187\right)$.

In an elevated plus maze test, Rapgef2-cKO mice preferred the open arms as judged by increased percentage of entries into the open arms (Fig. $2 \mathrm{i}, \mathrm{F}_{1,42}=8.453, p=0.0058$ ) and in the percentage of spent time in the open arms (Fig. 2j, $\mathrm{F}_{1,42}=6.229, p=0.0166$ ). On the other hand, Rapgef6-KO mice did not show any significant difference compared to wild-type mice in the open arms entry percentage and the percentage of spent time in the open arms (Fig. 2k, $\mathrm{F}_{1,38}=1.328, p=0.2564$; Fig. 2l, $\mathrm{F}_{1,38}=0.095$, $p=0.7597$, respectively).

\section{Effects on depression-like behavior}

To assess depression-like behavior, we employed the Porsolt forced swim test. We used the protocol in which the tests are performed in consecutive 2 days. This protocol, originally developed for the experiments using rats, is suitable for interpreting behavioral phenotypes more comprehensively. On day 1, Rapgef2-cKO mice did not show no significant genotype effect in the immobility (Fig. 3a, left, genotype effect, $\mathrm{F}_{1,43}=2.71, p=0.107$; genotype $\times$ time interaction, $\mathrm{F}_{9,387}=1.248, p=0.2639$ ). On day 2 , Rapgef2-cKO mice exhibited increased immobility during the 4-10 min time windows with an inverse trend seen during the 1-3 min time windows (Fig. 3a, right, genotype effect, $\mathrm{F}_{1,43}=0.065, p=0.8007$; genotype $\times$ time

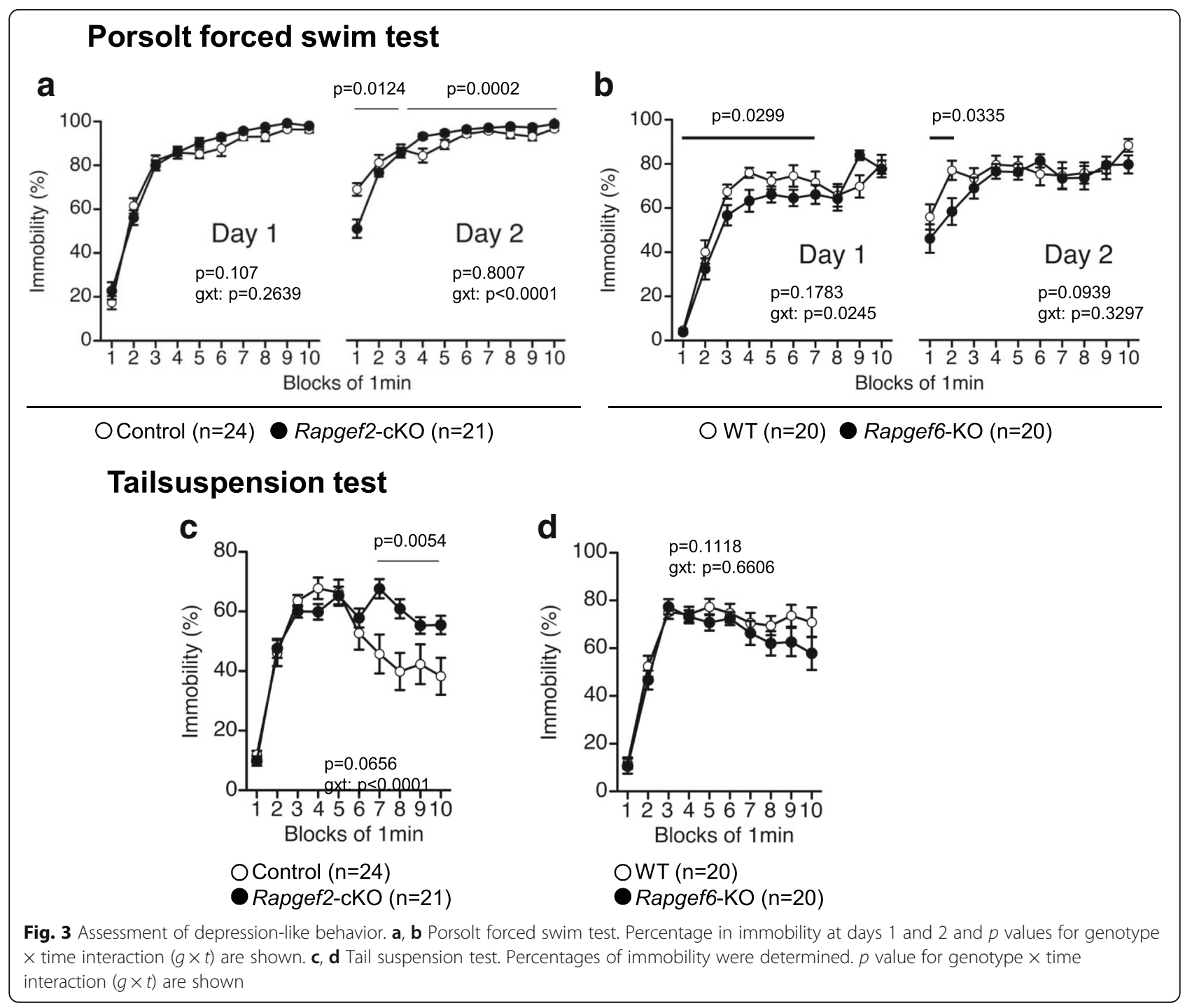


interaction, $\mathrm{F}_{9,387}=7.024, p<0.0001$; genotype effect, 1 3 min time windows, $\mathrm{F}_{1,43}=6.82, p=0.0124 ; 4-10 \mathrm{~min}$ time windows, $\mathrm{F}_{1,43}=17.252, p=0.0002$ ). In contrast, Rapgef6-KO mice exhibited decreased immobility during the first 1-7 $\mathrm{min}$ at day 1 (Fig. 3b, left, genotype effect, $\mathrm{F}_{1,38}=1.881, p=0.1783 ;$ genotype $\times$ time interaction, $\mathrm{F}_{9,342}=2.158, p=0.0245$; genotype effect, $1-7 \mathrm{~min}$ time windows, $\left.\mathrm{F}_{1,38}=5.089, p=0.0299\right)$. At day 2, Rapgef6-KO mice exhibited decreased immobility specifically in the initial phases of the test (Fig. 3b, right, genotype effect, $\mathrm{F}_{1,38}=2.952, p=0.0939 ;$ genotype $\times$ time interaction, $\mathrm{F}_{9,342}=1.146, p=0.3297$; genotype effect, $1-2 \mathrm{~min}$ time windows, $\mathrm{F}_{1,38}=4.865, p=0.0335$ ).

To further assess depression-like phenotype, we performed the tail suspension test. Rapgef2-cKO mice exhibited a significant increase in the percentage of immobility, during the 7-10 min time frame (Fig. 3c, genotype effect, $\mathrm{F}_{1,43}=3.569, p=0.0656 ;$ genotype $\times$ time interaction, $\mathrm{F}_{9,387}=4.481, p<0.0001 ; 7-10$ min time windows, $\mathrm{F}_{1,43}=$ $8.574, p=0.0054)$. In contrast, Rapgef6-KO mice did not exhibit any significant differences compared to wild-type mice (Fig. 3d, genotype effect, $\mathrm{F}_{1,38}=2.651, p=0.1118$; genotype $\times$ time interaction, $\mathrm{F}_{9,342}=0.752, p=0.6606$ ).

\section{Effects on startle response and prepulse inhibition test}

Alterations in RAPGEF2 and RAPGEF6 were reported in a group of schizophrenic patients [28, 29]. Therefore, we performed the prepulse inhibition test to investigate whether Rapgef2-cKO mice and Rapgef6-KO mice exhibited schizophrenia-like phenotype. Rapgef2-cKO mice and Rapgef6-KO mice significantly exhibited a decreased startle amplitude when tested with the auditory stimuli at the sound pressure levels of 110 and $120 \mathrm{~dB}$ (Fig. 4a, $\mathrm{F}_{1,43}=4.129, p=0.0484$; Fig. 4c, $\mathrm{F}_{1,38}=6.287, p=0.0166$, respectively). However, we failed to detect any significant effects of Rapgef2 or Rapgef6 deficiency in the prepulse inhibition test (Fig. 4b, $110 \mathrm{~dB}$ startle, $\mathrm{F}_{1,43}=1.161, p=0$. 2873; $120 \mathrm{~dB}$ startle, $\mathrm{F}_{1,43}=0.293, p=0.5912$; Fig. 4d, $110 \mathrm{~dB}$ startle, $\mathrm{F}_{1,38}=1.007, p=0.322 ; 120 \mathrm{~dB}$ startle, $\mathrm{F}_{1,38}=0.222, p=0.6404$, respectively).

\section{Effects on social behavior}

To assess the effects of Rapgef2 and Rapgef6 genotypes on social behavior, a social interaction test with novel environment was performed (Fig. 5a-j). Although the Rapgef2 genotype did not affect the total duration of contacts (Fig. 5a, $\mathrm{F}_{1,18}=0.048, p=0.8299$ ), Rapgef 2 -cKO mice exhibited significant increases in the number of contacts (Fig. 5b, $\mathrm{F}_{1,18}=28.989, p<0.0001$ ) and in the total duration of active contacts (Fig. $5 c, \mathrm{~F}_{1,18}=27.519$, $p<0.0001)$ accompanied by a decrease in the duration of each contact (Fig. $5 d, F_{1,18}=5.566, p=0.0298$ ). Further, the distance traveled was increased in Rapgef2-cKO mice (Fig. 5e, $\mathrm{F}_{1,18}=39.017, p<0.0001$ ). Rapgef6-KO mice exhibited behavior trends similar to those of Rapgef2-cKO mice. Even though the total duration of contacts was not affected (Fig. 5f, $\mathrm{F}_{1,18}=0.215, p=0$. 6484), Rapgef6-KO mice showed increases in the number of contacts (Fig. 5g, $\mathrm{F}_{1,18}=9.088, p=0.0074$ ) and in the total duration of active contacts (Fig. 5h, $\mathrm{F}_{1,18}=4$. $132, p=0.0571$ ) accompanied by a decrease in the duration of each contact (Fig. 5i, $\mathrm{F}_{1,18}=5.719, p=0.0279$ ). Additionally, the distance traveled was increased in Rapgef6-KO mice (Fig. 5j, $\mathrm{F}_{1,18}=6.421, p=0.0208$ ).

Next, a social interaction test using a home cage was performed (Fig. 5k-n). We did not observe any statistically significant alteration depending on the genotype of Rapgef2 or Rapgef6 in the mean number of particles formed with mice examined in each time window (Fig. 5k, throughout the experimental period, $\mathrm{F}_{1,16}=0.743, p=0$. 4016; light period, $\mathrm{F}_{1,16}=0.031, p=0.8628$; dark period, $\mathrm{F}_{1,16}=1.65, \quad p=0.2173 ; \quad$ Fig. $5 \mathrm{~m}$, throughout the

\section{Startle response/prepulse inhibition test}

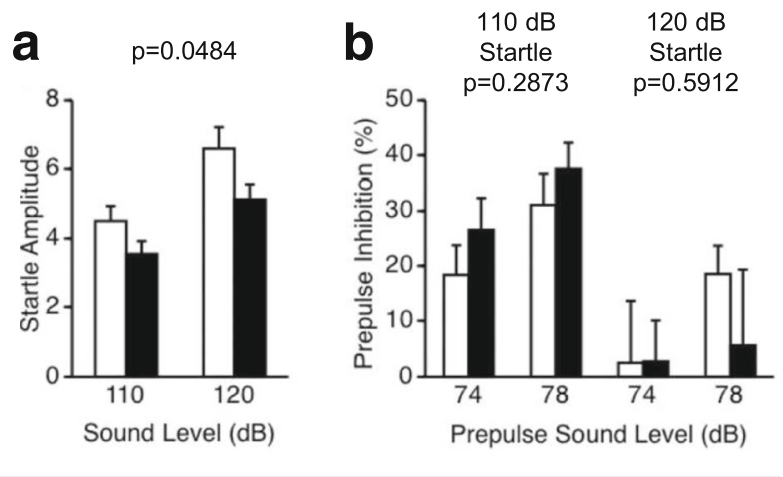

$\square$ Control (n=24) $\square$ Rapgef2-cKO (n=21)
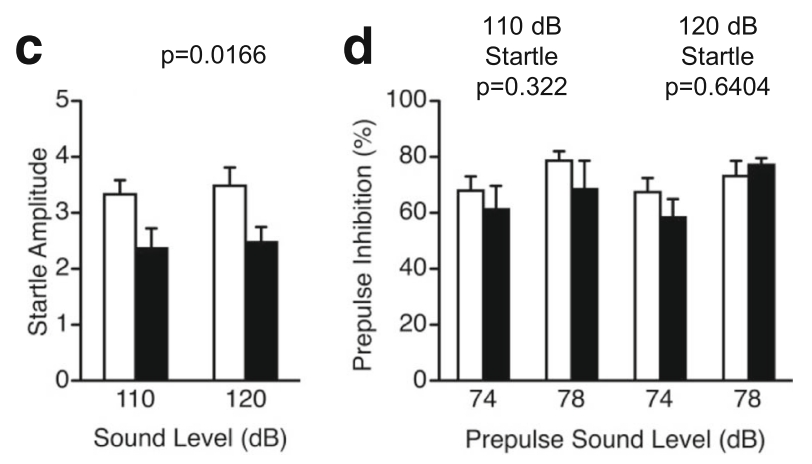

$\square \mathrm{WT}(\mathrm{n}=20)$

Rapgef6-KO ( $\mathrm{n}=20)$

Fig. 4 Startle response/prepulse inhibition test. Amplitudes of startle response $(\mathbf{a}, \mathbf{c})$ and percentages of prepulse inhibition (b, d) were determined 


\section{Social interaction test (Novel environment)}
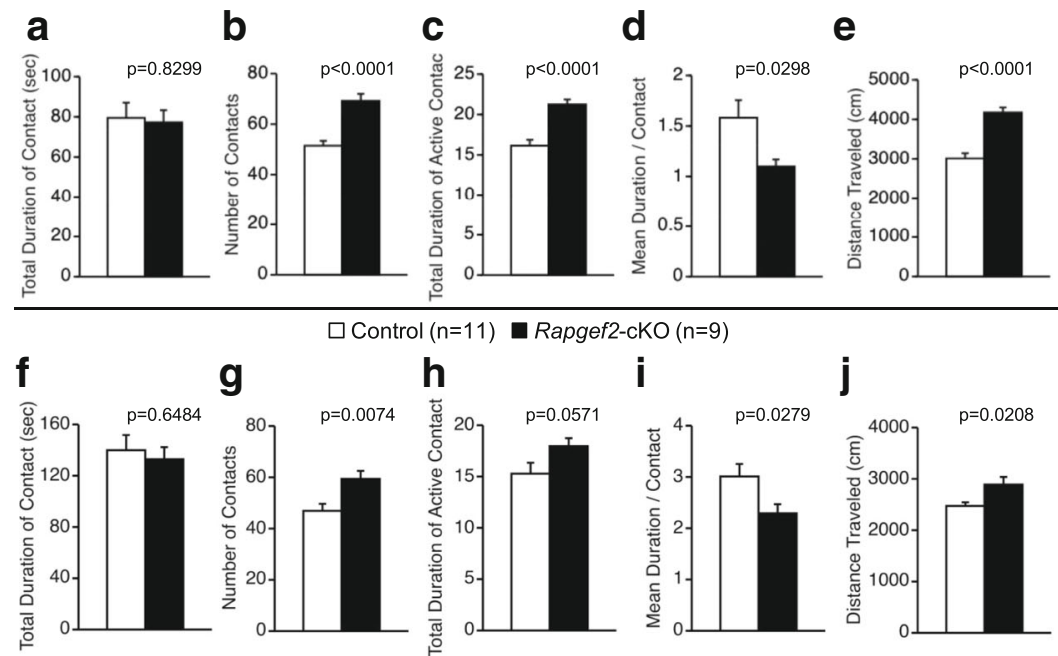

$\square W T(n=20)$ Rapgef6-KO $(n=20)$

\section{Social interaction test (Home cage)}

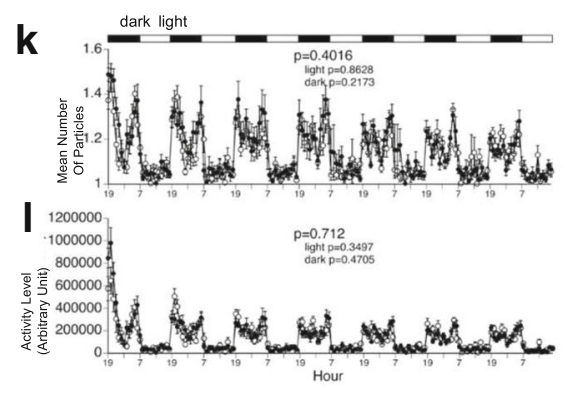

OControl $(n=10)$ Rapgef2-cKO $(n=8)$

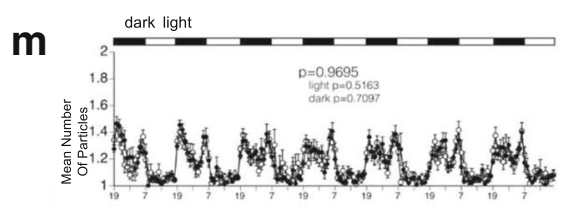

n 1000000

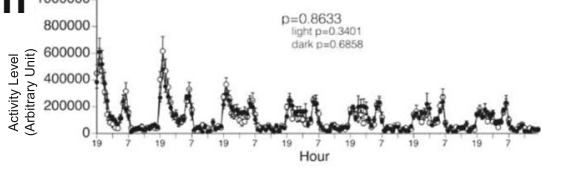

WT $(n=20)$ Rapgef6-KO $(n=20)$

\section{Crawley's sociability and social novelty preference test.}
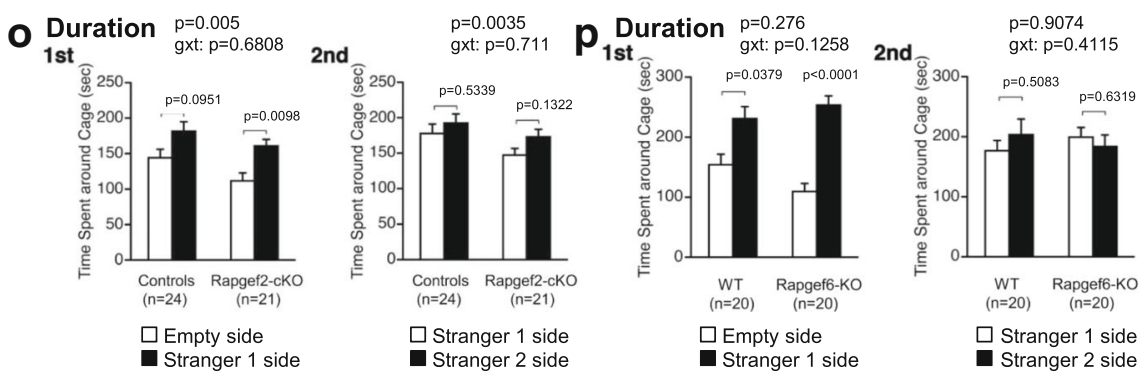

Fig. 5 Assessment for social behavior. $\mathbf{a}$-j Social interaction test in a novel environment. Total duration of contacts $(\mathbf{a}$, $\mathbf{f})$, the number of contacts $(\mathbf{b}, \mathbf{g})$, total duration of active contacts $(\mathbf{c}, \mathbf{h})$, mean duration of each contact $(\mathbf{d}, \mathbf{i})$, and total distance traveled $(\mathbf{e}, \mathbf{j})$ were determined. $\mathbf{k}-\mathbf{n}$ Social interaction test in a home cage. Means of the numbers of the particles formed with the mice tested were determined $(\mathbf{k}, \mathbf{m})$. The activity levels were calculated (I, n). o, p Crawley's sociability and social novelty preference test. Time spent around the indicated cages was determined. In 1st, a wire cage keeping a stranger mouse (Stranger 1 side) and an empty wire cage (Empty side) were used. In 2nd, a cage keeping a familiar mouse (Stranger 1 side) and a cage keeping another stranger mouse (Stranger 2 side) were used. $p$ values for genotype effects are shown on the top of each bargraph

experimental period, $\mathrm{F}_{1,18}=0.002, \quad p=0.9695 ;$ light period, $\mathrm{F}_{1,18}=0.438, p=0.5163$; dark period, $\mathrm{F}_{1,18}=0.143$, $p=0.7097$ ) or in the activity levels (Fig. 5l, throughout the experimental period, $\mathrm{F}_{1,16}=0.141, p=0.712$; light period, $\mathrm{F}_{1,16}=0.928, p=0.3497$; dark period, $\mathrm{F}_{1,16}=0.546$, $p=0.4705$; Fig. $5 n$, throughout the experimental period,
$\mathrm{F}_{1,18}=0.031, p=0.8633 ;$ light period, $\mathrm{F}_{1,18}=0.96, p=0$. 3401 ; dark period, $\left.\mathrm{F}_{1,18}=0.169, p=0.6858\right)$. These results seemed somewhat contradictory because hyperlocomotion phenotype in this test was not obvious in Rapgef2-cKO mice, which at the age of 18-24 weeks showed a marked increase in the locomotor activity in the open field test 
(Fig. 2a). Therefore, we performed the open field test using Rapgef2-cKO mice after the completion of the social interaction test, and found hyperlocomotion phenotype in aged (72-78 weeks old) Rapgef2-cKO mice (data not shown).

To further assess the social behavior, we performed Crawley's sociability and social novelty preference tests (Fig. 5o, p), a well-designed method to investigate the effects of complex genetics on sociability and preference for social novelty [36, 37]. First, we performed the sociability test using the test equipment containing an empty wire cage and a wire cage apparatus keeping a stranger mouse (Stranger 1). Rapgef2-cKO mice spent a longer time around the cage containing Stranger 1 than around the empty cage, but such trends were relatively weak in control mice (Fig. 5o, 1st, control mice, $t=1.741, \mathrm{df}=23, p=0.0951$; Rapgef2cKO mice, $t=2.856, \mathrm{df}=20, p=0.0098$, Stranger 1 side vs. empty side, paired $t$-test). A two-way repeated measures ANOVA demonstrated that Rapgef2-cKO mice spent significantly shorter time around the cages (Fig. 5o, 1st, genotype effect, $\mathrm{F}_{1,43}=8.754, p=0.005 ;$ genotype $\times$ cage interaction, $\mathrm{F}_{1,43}=0.172, p=0.6808$ ). We next performed the social novelty preference test using the test equipment containing a wire cage keeping another unfamiliar mouse (Stranger 2) and a wire cage keeping Stranger 1 that had already been used in the preceding trials and thus became familiar to the mice subjected to this test (Fig. 5o, 2nd). The time spent around the cage keeping Stranger 2 was not considerably different from the time spent around the cage keeping Stranger 1 regardless of the Rapgef2 genotype (Fig. 5o, 2nd, control mice, $t=0.622$, df $=23, p=0.5399$; Rapgef2-cKO mice, $t=1.569, \mathrm{df}=20, p=0.1322$, Stranger 2 side vs. Stranger 1side, paired $t$-test). A two-way repeated measures ANOVA again demonstrated that Rapgef2-cKO mice spent significantly shorter time around the cages (Fig. 5o, 2nd, genotype effect, $\mathrm{F}_{1,43}=9.539, p=0$. 0035 ; genotype $\times$ cage interaction, $\mathrm{F}_{1,43}=0.139, p=0.711$ ) .

We performed similar experiments to assess the effects of the Rapgef6 genotype. Rapgef6-KO mice spent longer time around the cage containing Stranger 1 than around the empty cage, and similar trends were detected in wild-type control mice (Fig. 5p, 1st, wildtype mice, $t=-2.231, \mathrm{df}=19, p=0.0379 ;$ Rapgef6-KO mice, $t=-5.607, \mathrm{df}=19, p<0.0001$, Stranger 1 side vs. empty side, paired $t$-test). A two-way repeated measures ANOVA demonstrated that no significant genotype effect was observed in the time spent around cage between wild-type mice and Rapgef6-KO mice (Fig. 5p, 1st, genotype effect, $\mathrm{F}_{1,38}=1.221, p=0.276$; genotype $\times$ cage interaction, $\left.\mathrm{F}_{1,38}=2.451, p=0.1258\right)$. In the social novelty preference test, the time spent around the cage keeping Stranger 2 was not significantly different from the time spent around the cage keeping Stranger 1 regardless of Rapgef6 genotype (Fig. $5 \mathrm{p}, 2 n d$, wild-type mice, $t$ $=-0.674, \mathrm{df}=19, p=0.5083 ;$ Rapgef6-KO mice, $t=0.487$, $\mathrm{df}=19, p=0.6319$, Stranger 2 side vs. Stranger 1 side, paired $t$-test). A two-way repeated measures ANOVA again demonstrated that no significant genotype effect was observed in the time spent around cage between wild-type mice and Rapgef6-KO mice (Fig. 5p, 2nd, genotype effect, $\mathrm{F}_{1,38}=0.014, p=0.9074$; genotype $\times$ cage interaction, $\mathrm{F}_{1,38}=0.689, p=0.4115$ ).

\section{Effects on cognitive function}

Effects of Rapgef2 or Rapgef6 deficiency on the spatial reference memory were assessed by the Barnes circular maze test. During acquisition tests, Rapgef2-cKO mice needed longer time (Fig. 6a, $\mathrm{F}_{1,43}=2.556, p=0.1172$ ), made more search errors (Fig. 6b, $\mathrm{F}_{1,43}=18.985, p<0.0001$ ) and traveled longer distances before reaching the correct target hole (Fig. $6 c, F_{1,43}=18.746, p<0.0001$ ), indicating that the learning performance was lower in Rapgef2-cKO mice. The probe trials using the maze from which an escape box was omitted were performed 1 day and 1 month after training. In the 1-day probe tests, Rapgef2-cKO mice needed longer time (Fig. 6d, $F_{1,43}=8.983, p=0.0045$ ), made a larger number of errors (Fig. 6e, $\mathrm{F}_{1,43}=13.605, p=0.0006$ ) and traveled longer distances (Fig. 6f, $\mathrm{F}_{1,43}=16.909, p=0.0002$ ) before getting to the correct target hole. Although genotype effect was not evident, a two-way repeated measures ANOVA indicated that there was a statistically significant interaction between the time spent around each hole and Rapgef2 genotype (Fig. 6g, genotype effect, $\mathrm{F}_{1,43}=0.062, p=0.8026$; genotype $\times$ target interaction, $\left.\mathrm{F}_{1,43}=9.572, p<0.0001\right)$. The accuracy of spatial memory in Rapgef2-cKO mice seemed to be worse than that of control mice (Fig. 6h, control mice, $t=3.552, \mathrm{df}=23, p=0.0017$; Rapgef2-cKO mice, $t=2.752, \quad \mathrm{df}=20, p=0.0123$, target vs. adjacent holes, paired $t$-test; genotype effect, $\mathrm{F}_{1,43}=23.594, p<0.0001$; genotype $\times$ target interaction, $\mathrm{F}_{1,43}=4.897, p=0.0323$, two-way repeated measures ANOVA). In the 1-month probe tests, although the latency to reach the correct target hole was not significantly affected (Fig. 6i, $F_{1,42}=0.488$, $p=0.4885)$, Rapgef2-cKO mice made significantly more errors (Fig. 6j, $F_{1,42}=9.308, p=0.0039$ ) and traveled longer distance (Fig. 6k, $\mathrm{F}_{1,42}=5.991, p=0.0186$ ) before arriving to the target hole. The Rapgef2 genotype significantly affected the time spent around the hole (Fig. 6l, genotype effect, $\left.\mathrm{F}_{1,42}=6.468, p=0.0148\right)$. The accuracy of spatial memory seemed to be worse in Rapgef2-cKO mice (Fig. $6 \mathrm{~m}$, control mice, $t=3.392$, $\mathrm{df}=23, p=0.0025$, Rapgef2-cKO mice, $t=0.433, \mathrm{df}=19, p=0.6696$, target holes vs. adjacent holes, paired $t$-test; genotype effect, $\mathrm{F}_{1,43}=0.317, p=0.5762$, genotype $\times$ target interaction, $\mathrm{F}_{1,43}=5.445, \quad p=0.0244$, two-way repeated measures ANOVA). In striking contrast, Rapgef6-KO mice did not exhibit any deficiency in the Barnes circular maze test. There were no significant differences in the latency to find the target hole (Fig. 6n, $\mathrm{F}_{1,38}=0.0004, p=0.9841$ ), the 

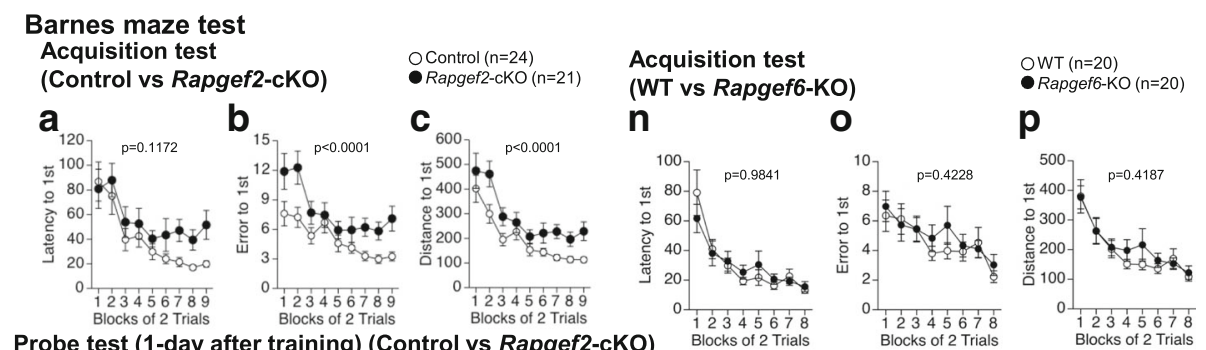

Probe test (1-day after training) (Control vs Rapgef2-cKO)
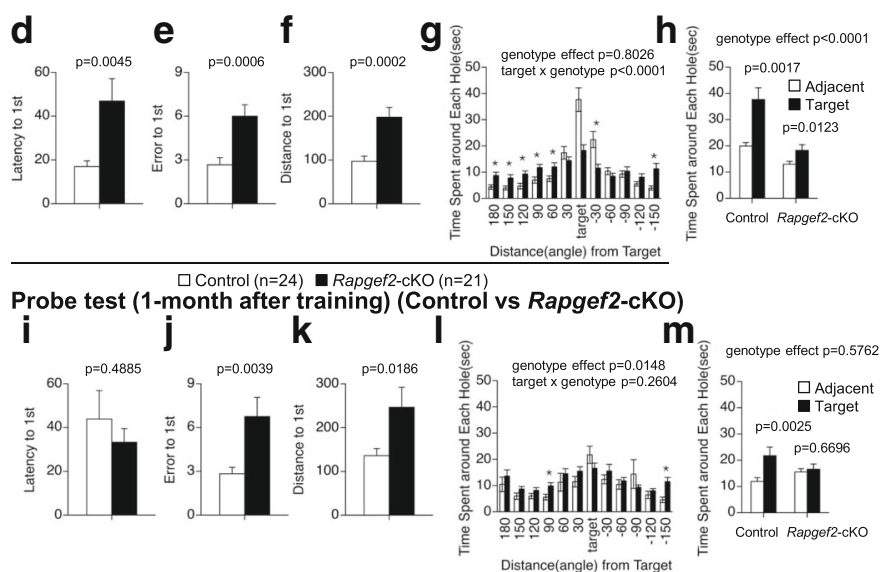

$\square$ Control (n=24) $\square$ Rapgef2-cKO ( $\mathrm{n}=21)$

Probe test (1-day after training) (WT vs Rapgef6-KO)

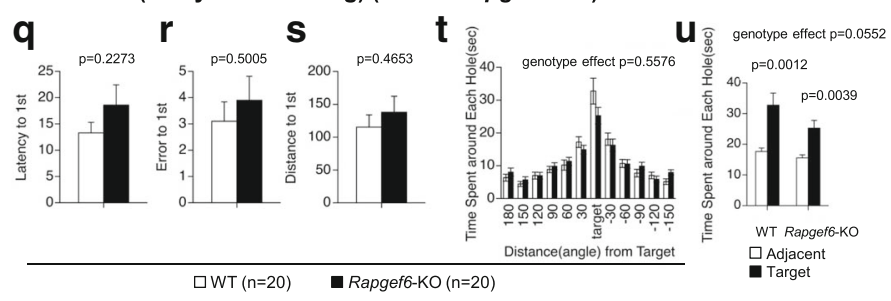

Probe test (1-month after training) (WT vs Rapgef6-KO)

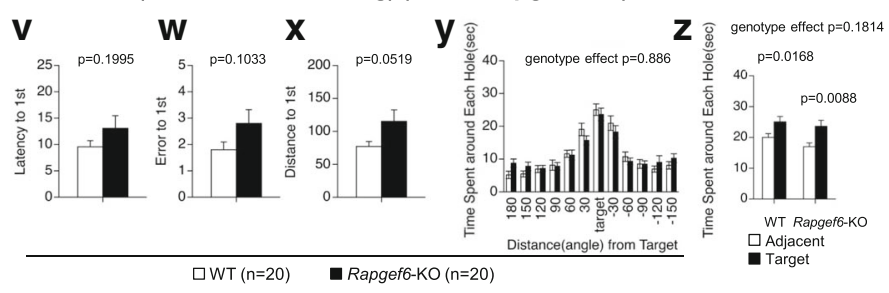

Fig. 6 Spatial learning and memory. a-m Effects of Rapgef2 deficiency. Data obtained with acquisition tests, where latency (a), number of errors made (b) and distance traveled (c) before acquisition of a target were determined in each block of 2 trials, are shown. Data obtained with probe test performed 1 day after training, where latency $(\mathbf{d})$, number of errors made (e), and distance traveled (f) before acquisition of the target were determined, time spent around each hole whose locations are indicated as "Distance from Target" in angle were determined (g), and time spent around the target and its adjacent holes was calculated $(\mathbf{h})$, are shown. Data obtained with probe test performed 1 month after training, where latency (i), number of errors made $(\mathbf{j})$, and distance traveled $(\mathbf{k})$ before acquisition of the target were determined, time spent around each hole whose locations are indicated as "Distance from Target" in angle were determined (I), and time spent around the target and its adjacent holes was calculated (m), are shown. $\mathbf{n}-\mathbf{z}$ Effects of Rapgef6 deficiency. Data obtained with acquisition test (n-p), probe test 1 day after training (q-u), and probe test 1 month after training $(\mathbf{v}-\mathbf{z})$, all of which were performed with methods equivalent to those for examining effects of Rapgef 2 deficiency, are shown. ${ }^{*}, p<0.05$

number of search errors made (Fig. 6o, $\mathrm{F}_{1,38}=0.657, p=0$. 4228), and the distance to reach the target hole (Fig. 6p, $\mathrm{F}_{1,38}=0.668, p=0.4187$ ) during acquisition. In the probe tests performed at both 1 day and 1 month after training, Rapgef6-KO mice did not show any significant difference compared to wild-type mice in the latency (1-day probe test, Fig. 6q, $\mathrm{F}_{1,38}=1.506, p=0.2273$; 1-month probe test, Fig. $\left.6 v, F_{1,38}=1.705, p=0.1995\right)$, the number of search errors (1-day probe test, Fig. 6r, $\mathrm{F}_{1,38}=0.463, p=0.5005$; 1 -month probe test, Fig. $6 \mathrm{w}, \mathrm{F}_{1,38}=2.786, p=0.1033$ ) and 
the distance (1-day probe test, Fig. $6 \mathrm{~s}, \mathrm{~F}_{1,38}=0.544, p=0$. 4653; 1-month probe test, Fig. $6 x, F_{1,38}=4.029, p=0$. 0519). No significant genotype effects were detected in the time spent around each holes (1-day probe test, Fig. 6t, genotype effect, $\mathrm{F}_{1,38}=0.35, p=0.5576 ; 1$-month probe test, Fig. 6y, genotype effect, $\left.\mathrm{F}_{1,38}=0.021, p=0.886\right)$ and the accuracy of spatial memory (1-day probe test, Fig. $6 \mathrm{u}$, genotype effect, $\mathrm{F}_{1,38}=0.3912, p=0.0552$; control mice, $t=3.79, \mathrm{df}=29, p=0.0012$; Rapgef6-KO mice, $t=3.282$, $\mathrm{df}=19, p=0.0039$, target vs. adjacent holes, paired $t$-test; 1 -month probe test, Fig. 6z, genotype effect, $F_{1,38}=1.853$, $p=0.1814$; control mice, $t=2.620, \mathrm{df}=19, p=0.0168$; Rapgef6-KO mice, $t=2.920, \mathrm{df}=19, p=0.0088$, target vs. adjacent holes, paired $t$-test).

We performed the T-maze test to assess the genotype effects on the working memory (Fig. 7). We employed a protocol for a forced alternation task test with food deprivation (for examining the effects of Rapgef2 deficiency) and that for a forced alternation task test without food deprivation (for examining the effects of Rapgef6 deficiency). These protocols are being widely used for the assessment of the working memory defects even though the level of exploring motivation, such as that associated with the presence or absence of reinforcement, is different among these two protocols. In both protocols, mice were subjected to four consecutive sessions to remember the locations of the arms that were previously visited. Rapgef2-cKO mice had a significantly decreased percentage of correct answers in the sessions 1 through 4 compared to control mice (Fig. $7 \mathrm{a}, \mathrm{F}_{1,43}=30.696, p<0$. 0001). In contrast, we failed to detect the effects of Rapgef6 deficiency on the percentage of correct answers in sessions 1 through 4 (Fig. $7 \mathrm{~b}, \mathrm{~F}_{1,38}=1.361, p=0.2506$ ). Thus, we performed the delayed alternation task test, which successfully detected a significant decrease in the percentage of correct answering in Rapgef6-KO mice (Fig. $7 \mathrm{c}, \mathrm{F}_{1,38}=7.996, p=0.0074$ ).

\section{Effects on fear-conditioned memory}

Fear-conditioned memory was assessed by a contextual and cued fear conditioning test. In Rapgef2-cKO mice, the percentage of the time of freezing caused by the foot-shock was significantly reduced during the conditioning phase (Fig. 8a, $\mathrm{F}_{1,43}=32.949, p<0.0001$ ). However, the distance traveled during electrical foot shocks was not significantly impacted by Rapgef2 genotype (Fig. 8d, foot shock 1, $\mathrm{F}_{1,43}=10.489, p=0.4883$; foot shock $2, \mathrm{~F}_{1,43}=0.423, p=0.5189$; foot shock $3, \mathrm{~F}_{1,43}=1.026$, $p=0.3168)$, suggesting that Rapgef2 deficiency had no effect on the sensitivity to the electric foot-shock. In the context testing performed at day 2 ( 1 day after the last conditioning), the percentage of the freezing time was significantly decreased in Rapgef2-cKO mice compared to control mice (Fig. 8b, left; $\mathrm{F}_{1,43}=36.049, p<0.0001$ ). Given the possibility that the percentage of freezing time could have been underestimated in Rapgef2-cKO mice due to their hyperlocomotion phenotype, we used an activity suppression ratio as a secondary index of fear in order to control baseline activity [38, 39]. In Rapgef2-cKO mice, the calculated activity suppression ratio was significantly increased (Fig. 8e, left, $\mathrm{F}_{1,43}=15.048, p=0.0004$ ). The cued testing with an altered context was then performed. Before the conditioned stimulus (CS; $55 \mathrm{~dB}$ white noise) was given, the percentage of the freezing time were significantly reduced in Rapgef2-cKO mice compared to that in control mice (Fig. 8b, right, during pre-CSperiod, $\mathrm{F}_{1,43}=4.641, p=0.0369$ ). There was an observed tendency that the percentage of the freezing time in Rapgef2cKO mice was reduced during stimulation with the CS (Fig. 8b, right, during CS, $\mathrm{F}_{1,43}=4.003, p=0.0517$ ).

\section{T-maze test (Forced alternation)}
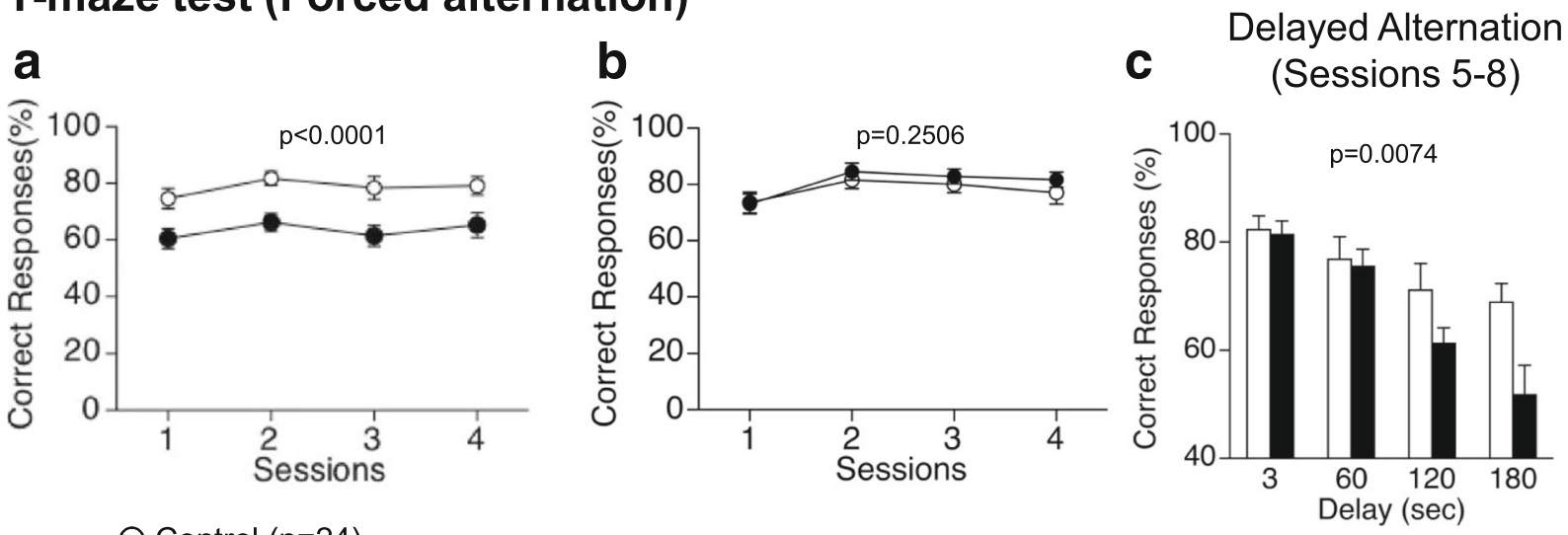

Control $(n=24)$
Rapgef2-cKO $(n=21)$

WT (n=20) Rapgef6-KO $(n=20)$

Fig. 7 T-maze test. Effects of Rapgef2 (a) or Rapgef6 (b, c) deficiency were examined 


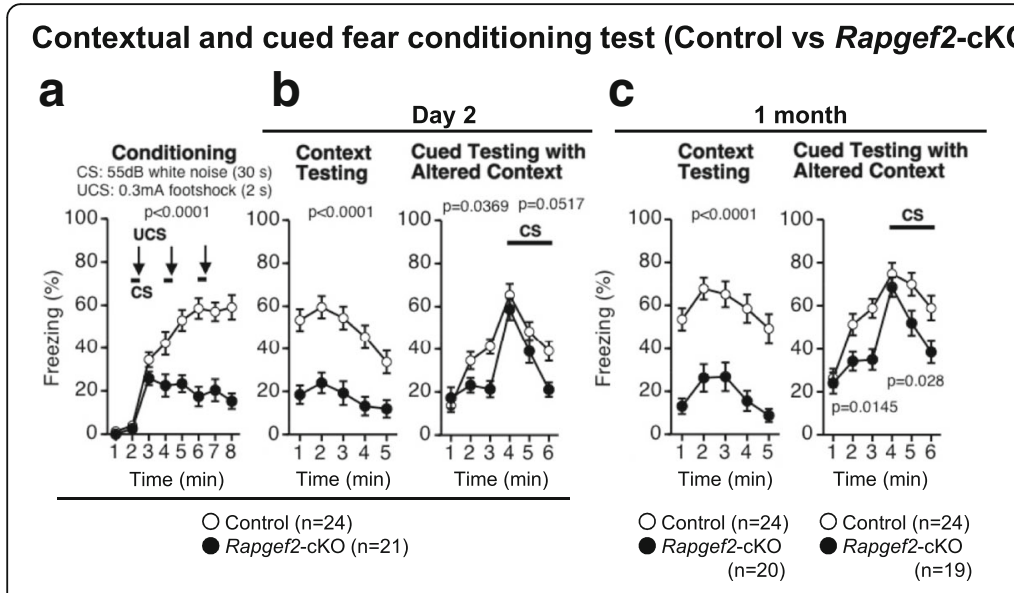

d

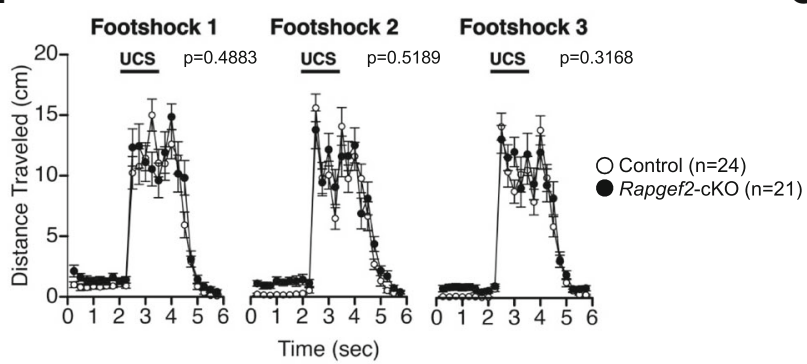

e

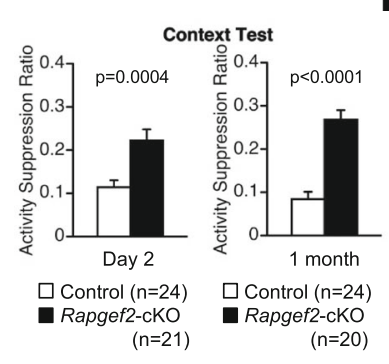

f

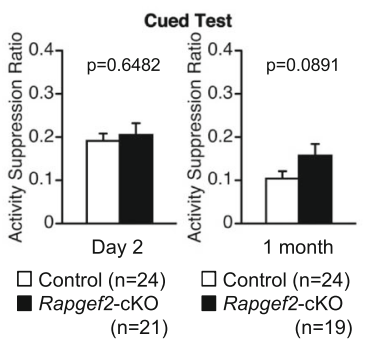

\section{Contextual and cued fear conditioning test (WT vs Rapgef6-KO)}

g

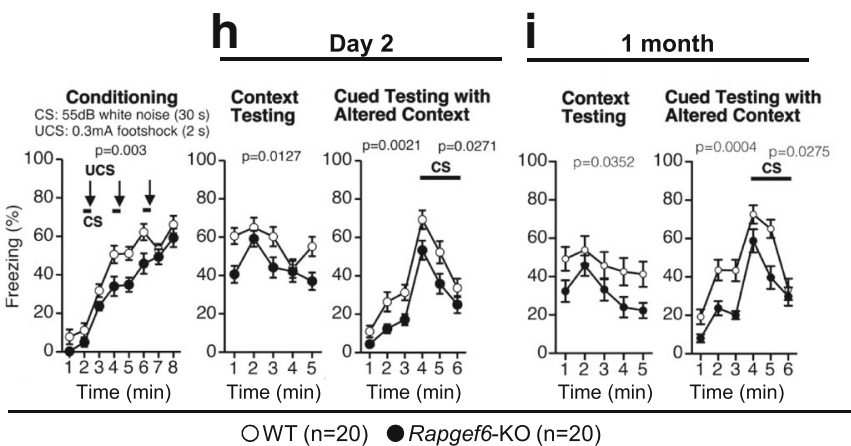

j

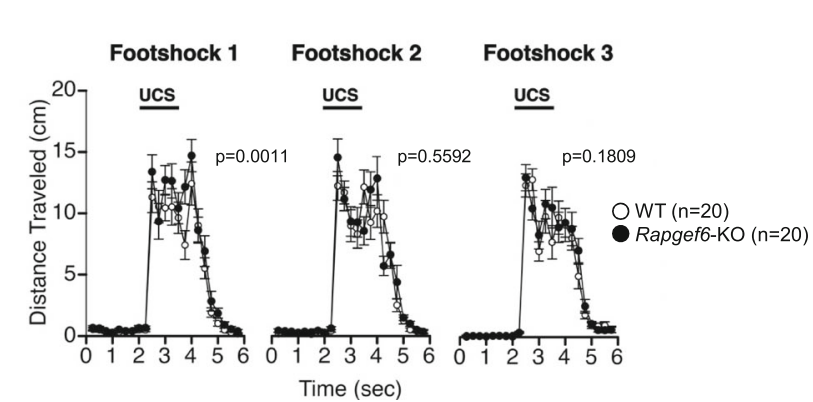

k
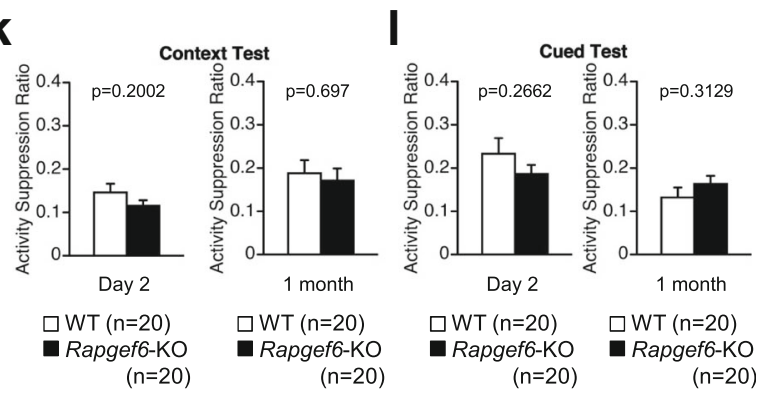

Fig. 8 Contextual and cued fear conditioning test. a-f Effects of Rapgef2 deficiency. Conditioned stimulus (CS; 55 dB white noise for 30 s) and aversive unconditioned stimulus (UCS; $0.3 \mathrm{~mA}$ foot shock for $2 \mathrm{~s}$ ) were given during the indicated periods. Percentages of freezing time during the conditioning phase (a), context testing and cued testing with altered context on day $2(\mathbf{b})$, and context testing and cued testing with altered context performed 1 month after the last conditioning $(\mathbf{c})$ were determined. Distance traveled in each time window of the conditioning phase was determined (d). Activity suppression ratios were determined for the context test (e) and cued test (f). $\mathbf{g}-\mathbf{I}$ Effects of Rapgef6 deficiency. The tests examining the effects of Rapgef6 deficiency were performed as in $\mathbf{a}-\mathbf{f}$, and percentages of freezing time ( $\mathbf{g}$-i), distance traveled in the conditioning phase $(\mathbf{j})$, and activity suppression ratios $(\mathbf{k}, \mathbf{I})$ were determined 
However, the activity suppression ratio during the CS was not significantly affected by Rapgef2 genotype (Fig. 8f, left, $\mathrm{F}_{1,43}=0.211, p=0.6482$ ). Similar tests conducted 1 month after the last conditioning revealed a reduction in the percentage of the freezing time in Rapgef2cKO mice (Fig. 8c). In the context test, Rapgef2-cKO mice exhibited a significant decrease in the percentage of freezing (Fig. 8c, left, $\mathrm{F}_{1,42}=36.559, p<0.0001$ ) as well as a significant increase in the activity suppuration ratio (Fig. 8e, right, $\left.\mathrm{F}_{1,42}=46.445, p<0.0001\right)$. In the cued test, the percentage of the freezing time was significantly reduced in Rapgef2-cKO mice regardless of the presence or absence of the CS (Fig. 8c, during pre-CS period, $\mathrm{F}_{1,41}=6.521$, $p=0.0145$; during CS, $\mathrm{F}_{1,41}=5.188, p=0.028$ ), but the activity suppression was not statistically affected by Rapgef 2 genotype (Fig. 8f, right, $\mathrm{F}_{1,41}=3.032, p=0.0891$ ). Next, similar experiments were carried out with Rapgef6-KO mice. In the conditioning phase, the percentage of the time of freezing caused by the foot-shock was significantly reduced in Rapgef6-KO mice (Fig. 8g, $\mathrm{F}_{1,38}=10.046$, $p=0.003)$. There was a significant difference in distance traveled during first electrical foot shock between Rapgef6$\mathrm{KO}$ and wild-type mice (Fig. 8j, foot shock $1, \mathrm{~F}_{1,38}=12.428$, $p=0.0011$; foot shock $2, \mathrm{~F}_{1,38}=0.347, p=0.5592$; foot shock $\left.3, \mathrm{~F}_{1,38}=1.858, p=0.1809\right)$. In the context testing performed at day 2 ( 1 day after the last conditioning), the percentage of the freezing time was decreased in Rapgef6KO mice compared to wild-type mice (Fig. 8h, left; $\left.\mathrm{F}_{1,38}=6.837, p=0.0127\right)$. However, the activity suppression ratio was not significantly increased, suggesting underestimation of the percentage of the freezing time probably due to the hyperlocomotion phenotype (Fig. 8k, left, $\mathrm{F}_{1,38}=1.699, p=0.2002$ ). The cued testing with an altered context was then performed. Before the conditioned stimulus (CS; $55 \mathrm{~dB}$ white noise) was given, the percentage of the freezing time was significantly reduced in Rapgef6-KO mice compared to that in wild-type mice (Fig. 8h, right, during pre-CS period, $\mathrm{F}_{1,38}=10.947$, $p=0.0021$ ). Further, in Rapgef6-KO mice, we observed a reduced percentage of the freezing time during stimulation with the CS (Fig. 8h, right, during CS, $\mathrm{F}_{1,38}=5.287$, $p=0.0271$ ). However, the activity suppression ratio during the CS was not significantly affected by the Rapgerf6 genotype (Fig. 8l, left, $\mathrm{F}_{1,38}=1.274, p=0$. 2662). Similar tests conducted 1 month after the last conditioning showed similar reduction in the percentage of the freezing time (Fig. 8i). In the context test, Rapgef6-KO mice exhibited a decrease in the percentage of freezing (Fig. 8i, left, $\mathrm{F}_{1,38}=4.772, p=0.0352$ ), but the activity suppression was not statistically affected by the Rapgef6 genotype (Fig. 8k, right, $\mathrm{F}_{1,38}=0.154, p=0.697$ ). In the cued test, the percentage of the freezing time was significantly reduced in Rapgef6-KO mice regardless of the presence or absence of the CS (Fig. 8i, during
pre-CS period, $\mathrm{F}_{1,38}=15.314, p=0.0004$; during CS, $\left.\mathrm{F}_{1,38}=5.254, p=0.0275\right)$, but the activity suppression was not statistically affected by Rapgef6 genotype (Fig. 81, right, $\mathrm{F}_{1,38}=1.046, p=0.3129$ ).

\section{Discussion and conclusions}

In this study, we performed a comprehensive battery of behavioral tests using mice deficient in Rapgef2 or Rapgef6 to gain insights into the role of Rapgef2 and Rapgef6 in higher brain functions in mammals. Results obtained in this study are summarized in Table 1. Our results revealed that Rapgef 2 deficiency had more drastic impact on the mice behavior than Rapgef6 deficiency. This could be partly attributed to the structural abnormalities evident in the brains of Rapgef2-cKO mice, including the interruption of pyramidal cells in the hippocampal CA1 region, CC agenesis, ECM, and enlarged lateral ventricles [24-26]. Indeed, such alterations in the brain structure have been implicated in a variety of disorders of higher brain functions. For instance, the hippocampus is important for learning and memory recalling [40], and the interhemispheric connections, including those with the CC, play an important role in coordination of the activities of each hemisphere [41], thereby contributing to the regulation of a variety of brain functions, such as memory fix or maintenance, memory recall, and spatial memory formation [42-45].

The open field test clearly detected hyperlocomotion phenotype in Rapgef2-cKO mice (Fig. 2a, b). This hyperlocomotion phenotype in Rapgef2 mutant mice seemed not to be affected by aging because it was detected both in younger and elder Rapgef2-cKO mice. In contrast, although Rapgef6-KO mice did not clearly exhibit difference in the total distance traveled, the Rapgef6 genotype significantly affected these indices in a time-dependent manner (Fig. 2c, genotype $\times$ time interaction, $F_{23,874}=2.350$, $p=0.0004)$. Even though the degree of the phenotypic changes in the locomotion was greater in Rapgef2-cKO mice than in Rapgef6-KO mice, the hyperlocomotion phenotypes appeared to be detected also in other tests performed in this study and seemed to affect the outputs of the behavioral tests. Thus, their hyperlocomotion phenotypes needed to be considered as confounding factors that should be paid particular attention upon the interpretation of data obtained here.

In Rapgef2-cKO mice, we detected depression-like phenotypes in the tail suspension test (Fig. 3c), where depression-like behavior was judged as increased immobility. Rapgef2-cKO mice exhibited depression-like phenotypes also in the porsolt forced swim test on day2, where immobility that could be associated with depression was increased in the 4-10 min windows (Fig. 3a, right). On the other hand, a decrease in the immobility detected in the 1-3 min windows seemed to be 
Table 1 Phenotype of Rapgef2-cKO and Rapgef6-KO mice in comprehensive behavioral test battery

\begin{tabular}{|c|c|c|c|c|c|c|c|}
\hline \multirow[t]{2}{*}{ Task } & \multirow[t]{2}{*}{ Test } & \multirow[t]{2}{*}{ Measure } & \multirow{2}{*}{$\begin{array}{l}\text { Rapgef2-cKO } \\
\text { (vs. Non-cKO) }\end{array}$} & \multirow{2}{*}{$\begin{array}{l}\text { Rapgef6-KO } \\
\text { (vs. WT) }\end{array}$} & \multicolumn{2}{|c|}{ Statistical analysis } & \multirow[t]{2}{*}{ Figure } \\
\hline & & & & & Rapgef2-cKO & Rapgef6-KO & \\
\hline \multirow[t]{4}{*}{$\begin{array}{l}\text { Physical } \\
\text { characteristics }\end{array}$} & Body weight & Weight (g) & n.s. & -- & $\begin{array}{l}F_{1,43}=1.745 \\
p=0.1935\end{array}$ & $\begin{array}{l}F_{1,38}=12.541 \\
p=0.0011\end{array}$ & Fig. 1a, e \\
\hline & Body temperature & Temperature $\left({ }^{\circ} \mathrm{C}\right)$ & n.s. & n.s. & $\begin{array}{l}F_{1,43}=1.991 \\
p=0.1654\end{array}$ & $\begin{array}{l}F_{1,38}=4.036 \\
p=0.0517\end{array}$ & Fig. $1 b, f$ \\
\hline & Grip strength (N) & Strength (N) & n.s. & n.s. & $\begin{array}{l}F_{1,43}=1.149 \\
p=0.2897\end{array}$ & $\begin{array}{l}F_{1,38}=0.062 \\
p=0.8053\end{array}$ & Fig. $1 c, g$ \\
\hline & Wire hang latency (s) & Latency (s) & -- & n.s. & $\begin{array}{l}F_{1,43}=16.785 \\
p=0.0002\end{array}$ & $\begin{array}{l}F_{1,38}=0.04 \\
p=0.8425\end{array}$ & Fig. $1 d, h$ \\
\hline Motor coordination & Rotarod & Latency to fall (s) & n.s. & n.s. & $\begin{array}{l}F_{1,43}=1.251 \\
p=0.2695\end{array}$ & $\begin{array}{l}F_{1,38}=0.708 \\
p=0.4052\end{array}$ & Fig. 1i, j \\
\hline Pain sensitivity & Hot plate & Latency (s) & - & n.s. & $\begin{array}{l}F_{1,43}=5.043 \\
p=0.0299\end{array}$ & $\begin{array}{l}F_{1,38}=1.045 \\
p=0.3131\end{array}$ & Fig. 1k, I \\
\hline $\begin{array}{l}\text { Exploratory } \\
\text { locomotion }\end{array}$ & Open field test & Distance traveled $(\mathrm{cm})$ & +++ & + (initial phase) & $\begin{array}{l}F_{1,43}=36.348 \\
p<0.0001\end{array}$ & $\begin{array}{l}F_{1,38}=0.03 \\
p=0.8638\end{array}$ & Fig. 2a, c \\
\hline \multirow[t]{5}{*}{$\begin{array}{l}\text { Anxiety-like } \\
\text { behavior }\end{array}$} & Open field test & Center time (s) & +++ & + (initial phase) & $\begin{array}{l}F_{1,43}=19.658 \\
p<0.0001\end{array}$ & $\begin{array}{l}F_{1,38}=0.002 \\
p=0.9656\end{array}$ & Fig. 2b, d \\
\hline & $\begin{array}{l}\text { Light/dark transition } \\
\text { test }\end{array}$ & Number of transitions & n.s. & n.s. & $\begin{array}{l}F_{1,43}=0.735 \\
p=0.3961\end{array}$ & $\begin{array}{l}F_{1,38}=0.395 \\
p=0.5335\end{array}$ & Fig. 2e, g \\
\hline & & $\begin{array}{l}\text { Latency to light } \\
\text { chamber (s) }\end{array}$ & n.s. & n.s. & $\begin{array}{l}F_{1,43}=0.501 \\
p=0.4827\end{array}$ & $\begin{array}{l}F_{1,38}=0.669 \\
p=0.4187\end{array}$ & Fig. 2f, h \\
\hline & $\begin{array}{l}\text { Elevated plus maze } \\
\text { test }\end{array}$ & $\begin{array}{l}\text { Entries into } \\
\text { open arms (\%) }\end{array}$ & ++ & n.s. & $\begin{array}{l}F_{1,42}=8.453 \\
p=0.0058\end{array}$ & $\begin{array}{l}F_{1,38}=1.328 \\
p=0.2564\end{array}$ & Fig. 2i, k \\
\hline & & Time on open arms (\%) & + & n.s. & $\begin{array}{l}F_{1,42}=6.229 \\
p=0.0166\end{array}$ & $\begin{array}{l}F_{1,38}=0.095 \\
p=0.7597\end{array}$ & Fig. $2 \mathrm{j}, \mathrm{I}$ \\
\hline \multirow[t]{3}{*}{$\begin{array}{l}\text { Behavioral } \\
\text { despair }\end{array}$} & $\begin{array}{l}\text { Porsolt forced swim } \\
\text { test }\end{array}$ & $\begin{array}{l}\text { Immobility (\%) } \\
\text { on Day } 1\end{array}$ & n.s. & $-(1-7 \min )$ & $\begin{array}{l}F_{1,43}=2.71 \\
p=0.107\end{array}$ & $\begin{array}{l}\mathrm{F}_{1,38}=1.881 \\
p=0.1783 \\
1-7 \mathrm{~min} \\
\mathrm{~F}_{1,38}=5.089 \\
p=0.0299\end{array}$ & Fig. 3a, b \\
\hline & & $\begin{array}{l}\text { Immobility (\%) } \\
\text { on Day } 2\end{array}$ & $\begin{array}{l}-(1-3 \mathrm{~min}) \\
++(4-10 \mathrm{~min})\end{array}$ & $-(1-2 \min )$ & $\begin{array}{l}F_{1,43}=0.065 \\
p=0.8007 \\
1-3 \text { min } \\
F_{1,43}=6.82 \\
p=0.0124 \\
4-10 \mathrm{~min} \\
F_{1,43}=17.252 \\
p=0.0002\end{array}$ & $\begin{array}{l}F_{1,38}=2.952 \\
p=0.0939 \\
1-2 \text { min } \\
F_{1,38}=4.865 \\
p=0.0335\end{array}$ & Fig. 3a, b \\
\hline & Tail suspension test & Immobility (\%) & $++(7-10 \mathrm{~min})$ & n.s. & $\begin{array}{l}\mathrm{F}_{1,43}=3.569 \\
p=0.0656 \\
7-10 \mathrm{~min} \\
\mathrm{~F}_{1,43}=8.574 \\
p=0.0054\end{array}$ & $\begin{array}{l}F_{1,38}=2.651 \\
p=0.1118\end{array}$ & Fig. $3 c, d$ \\
\hline \multirow[t]{3}{*}{$\begin{array}{l}\text { Sensorimotor } \\
\text { gating }\end{array}$} & Startle response test & Startle response & - & - & $\begin{array}{l}F_{1,43}=4.129 \\
p=0.0484\end{array}$ & $\begin{array}{l}F_{1,38}=6.287 \\
p=0.0166\end{array}$ & Fig. 4a, c \\
\hline & $\begin{array}{l}\text { Prepulse inhibition } \\
\text { test }\end{array}$ & $\begin{array}{l}\text { PPI (startle stimulus, } \\
110 \mathrm{~dB} \text { ) }\end{array}$ & n.s. & n.s. & $\begin{array}{l}F_{1,43}=1.161 \\
p=0.2873\end{array}$ & $\begin{array}{l}F_{1,38}=1.007 \\
p=0.322\end{array}$ & Fig. $4 b, d$ \\
\hline & & $\begin{array}{l}\text { PPI (startle stimulus, } \\
120 \mathrm{~dB} \text { ) }\end{array}$ & n.s. & n.s. & $\begin{array}{l}F_{1,43}=0.293 \\
p=0.5912\end{array}$ & $\begin{array}{l}F_{1,38}=0.222 \\
p=0.6404\end{array}$ & Fig. $4 b, d$ \\
\hline \multirow[t]{3}{*}{ Social interaction } & Novel environment & $\begin{array}{l}\text { Total duration of } \\
\text { contacts (s) }\end{array}$ & n.s. & n.s. & $\begin{array}{l}F_{1,18}=0.048 \\
p=0.8299\end{array}$ & $\begin{array}{l}F_{1,18}=0.215 \\
p=0.6484\end{array}$ & Fig. 5a, f \\
\hline & & $\begin{array}{l}\text { Number of } \\
\text { contacts }\end{array}$ & +++ & ++ & $\begin{array}{l}F_{1,18}=28.989 \\
p<0.0001\end{array}$ & $\begin{array}{l}F_{1,18}=9.088 \\
p=0.0074\end{array}$ & Fig. 5b, g \\
\hline & & $\begin{array}{l}\text { Total duration of } \\
\text { active contacts (s) }\end{array}$ & +++ & n.s. & $\begin{array}{l}F_{1,18}=27.519 \\
p<0.0001\end{array}$ & $\begin{array}{l}F_{1,18}=4.132 \\
p=0.0571\end{array}$ & Fig. $5 c, h$ \\
\hline
\end{tabular}


Table 1 Phenotype of Rapgef2-cKO and Rapgef6-KO mice in comprehensive behavioral test battery (Continued)

\begin{tabular}{|c|c|c|c|c|c|c|c|}
\hline \multirow[t]{2}{*}{ Task } & \multirow[t]{2}{*}{ Test } & \multirow[t]{2}{*}{ Measure } & \multirow{2}{*}{$\begin{array}{l}\text { Rapgef2-cKO } \\
\text { (vs. Non-cKO) }\end{array}$} & \multirow{2}{*}{$\begin{array}{l}\text { Rapgef6-KO } \\
\text { (vs. WT) }\end{array}$} & \multicolumn{2}{|c|}{ Statistical analysis } & \multirow[t]{2}{*}{ Figure } \\
\hline & & & & & Rapgef2-cKO & Rapgef6-KO & \\
\hline & & $\begin{array}{l}\text { Mean duration } \\
\text { of contact (s) }\end{array}$ & & & $\begin{array}{l}F_{1,18}=5.566 \\
p=0.0298\end{array}$ & $\begin{array}{l}F_{1,18}=5.719 \\
p=0.0279\end{array}$ & \\
\hline & & $\begin{array}{l}\text { Distance } \\
\text { traveled }(\mathrm{cm})\end{array}$ & +++ & + & $\begin{array}{l}F_{1,18}=39.017 \\
p<0.0001\end{array}$ & $\begin{array}{l}F_{1,18}=6.421 \\
p=0.0208\end{array}$ & Fig. $5 e, j$ \\
\hline & Home cage & $\begin{array}{l}\text { Mean number } \\
\text { of particles }\end{array}$ & n.s. & n.s. & $\begin{array}{l}\mathrm{F}_{1,16}=0.743 \\
p=0.4016 \\
\text { light period, } \\
\mathrm{F}_{1,16}=0.031 \\
p=0.8628 \\
\text { dark period, } \\
\mathrm{F}_{1,16}=1.65 \\
p=0.2173\end{array}$ & $\begin{array}{l}\mathrm{F}_{1,18}=0.002 \\
p=0.9695 \\
\text { light period, } \\
\mathrm{F}_{1,18}=0.438 \\
p=0.5163 \\
\text { dark period } \\
\mathrm{F}_{1,18}=0.143 \\
p=0.7097\end{array}$ & Fig. $5 k, m$ \\
\hline & & Activity levels & n.s. & n.s. & $\begin{array}{l}\mathrm{F}_{1,16}=0.141 \\
p=0.712 ; \\
\text { light period, } \\
\mathrm{F}_{1,16}=0.928 \\
p=0.3497 \\
\text { dark period, } \\
\mathrm{F}_{1,16}=0.546 \\
p=0.4705\end{array}$ & $\begin{array}{l}\mathrm{F}_{1,18}=0.031 \\
p=0.8633 \\
\text { light period, } \\
\mathrm{F}_{1,18}=0.96 \\
p=0.3401 \\
\text { dark period, } \\
\mathrm{F}_{1,18}=0.169 \\
p=0.6858\end{array}$ & Fig. 5l, n \\
\hline & Crawley's version & $\begin{array}{l}\text { Time spent around } \\
\text { cage (s) on } 15 t\end{array}$ & -- & n.s. & $\begin{array}{l}\mathrm{F}_{1,43}=8.754 \\
p=0.005\end{array}$ & $\begin{array}{l}F_{1,38}=1.221 \\
p=0.276\end{array}$ & Fig. 5o, p \\
\hline & & $\begin{array}{l}\text { Time spent around } \\
\text { cage (s) on 2nd }\end{array}$ & -- & n.s. & $\begin{array}{l}F_{1,43}=9.539 \\
p=0.0035\end{array}$ & $\begin{array}{l}F_{1,38}=0.014 \\
p=0.9074\end{array}$ & Fig. 5o, p \\
\hline \multirow[t]{14}{*}{ Spatial memory } & Barnes maze test & & & & & & \\
\hline & Acquisition test & Latency to 1st (s) & n.s. & n.s. & $\begin{array}{l}F_{1,43}=2.556 \\
p=0.1172\end{array}$ & $\begin{array}{l}F_{1,38}=0.0004 \\
p=0.9841\end{array}$ & Fig. 6a, n \\
\hline & & Error to $1 \mathrm{st}$ & +++ & n.s. & $\begin{array}{l}F_{1,43}=18.985, \\
p<0.0001\end{array}$ & $\begin{array}{l}F_{1,38}=0.657 \\
p=0.4228\end{array}$ & Fig. 6b, o \\
\hline & & Distance to 1st (mm) & +++ & n.s. & $\begin{array}{l}F_{1,43}=18.746 \\
p<0.0001\end{array}$ & $\begin{array}{l}F_{1,38}=0.668 \\
p=0.4187\end{array}$ & Fig. $6 c, p$ \\
\hline & $\begin{array}{l}\text { Probe test (1-day } \\
\text { after training) }\end{array}$ & Latency to 1st (s) & ++ & n.s. & $\begin{array}{l}F_{1,43}=8.983 \\
p=0.0045\end{array}$ & $\begin{array}{l}F_{1,38}=1.506 \\
p=0.2273\end{array}$ & Fig. $6 \mathrm{~d}, \mathrm{q}$ \\
\hline & & Error to $1 \mathrm{st}$ & ++ & n.s. & $\begin{array}{l}F_{1,43}=13.605 \\
p=0.0006\end{array}$ & $\begin{array}{l}F_{1,38}=0.463 \\
p=0.5005\end{array}$ & Fig. 6e, r \\
\hline & & Distance to $1 \mathrm{st}(\mathrm{mm})$ & ++ & n.s. & $\begin{array}{l}F_{1,43}=16.909 \\
p=0.0002\end{array}$ & $\begin{array}{l}F_{1,38}=0.544 \\
p=0.4653\end{array}$ & Fig. $6 f, s$ \\
\hline & & $\begin{array}{l}\text { Time spent around } \\
\text { each hole (s) }\end{array}$ & n.s. & n.s. & $\begin{array}{l}F_{1,43}=0.062 \\
p=0.8026\end{array}$ & $\begin{array}{l}F_{1,38}=0.35 \\
p=0.5576\end{array}$ & Fig. 6g, t \\
\hline & & $\begin{array}{l}\text { Time spent around } \\
\text { target hole (s) }\end{array}$ & --- & n.s. & $\begin{array}{l}F_{1,43}=23.594 \\
p<0.0001\end{array}$ & $\begin{array}{l}F_{1,38}=0.3912 \\
p=0.0552\end{array}$ & Fig. 6h, u \\
\hline & $\begin{array}{l}\text { Probe test (1-month } \\
\text { after training) }\end{array}$ & Latency to 1st (s) & n.s. & n.s. & $\begin{array}{l}F_{1,42}=0.488 \\
p=0.4885\end{array}$ & $\begin{array}{l}F_{1,38}=1.705 \\
p=0.1995\end{array}$ & Fig. 6i, v \\
\hline & & Error to $1 \mathrm{st}$ & ++ & n.s. & $\begin{array}{l}F_{1,42}=9.308 \\
p=0.0039\end{array}$ & $\begin{array}{l}F_{1,38}=2.786 \\
p=0.1033\end{array}$ & Fig. 6j, w \\
\hline & & Distance to 1st (mm) & + & n.s. & $\begin{array}{l}F_{1,42}=5.991 \\
p=0.0186\end{array}$ & $\begin{array}{l}F_{1,38}=4.029 \\
p=0.0519\end{array}$ & Fig. 6k, $x$ \\
\hline & & $\begin{array}{l}\text { Time spent around } \\
\text { each hole (s) }\end{array}$ & n.s. & n.s. & $\begin{array}{l}F_{1,42}=6.468 \\
p=0.0148\end{array}$ & $\begin{array}{l}F_{1,38}=0.021 \\
p=0.886\end{array}$ & Fig. 6l, y \\
\hline & & $\begin{array}{l}\text { Time spent around } \\
\text { target hole (s) }\end{array}$ & n.s. & n.s. & $\begin{array}{l}F_{1,43}=0.317 \\
p=0.5762\end{array}$ & $\begin{array}{l}F_{1,38}=1.853 \\
p=0.1814\end{array}$ & Fig. $6 \mathrm{~m}, \mathrm{z}$ \\
\hline Working memory & T-maze test & Correct responses (\%) & --- & $\begin{array}{l}\text { - - (delayed } \\
\text { alternation) }\end{array}$ & $\begin{array}{l}F_{1,43}=30.696 \\
p<0.0001\end{array}$ & $\begin{array}{l}\text { Session } 1-4 \\
F_{1,38}=1.361 \\
p=0.2506\end{array}$ & Fig. 7a, c \\
\hline
\end{tabular}


Table 1 Phenotype of Rapgef2-cKO and Rapgef6-KO mice in comprehensive behavioral test battery (Continued)

\begin{tabular}{|c|c|c|c|c|c|c|c|}
\hline \multirow[t]{2}{*}{ Task } & \multirow[t]{2}{*}{ Test } & \multirow[t]{2}{*}{ Measure } & \multirow{2}{*}{$\begin{array}{l}\text { Rapgef2-cKO } \\
\text { (vs. Non-cKO) }\end{array}$} & \multirow{2}{*}{$\begin{array}{l}\text { Rapgef6-KO } \\
\text { (vs. WT) }\end{array}$} & \multicolumn{2}{|c|}{ Statistical analysis } & \multirow[t]{2}{*}{ Figure } \\
\hline & & & & & Rapgef2-cKO & Rapgef6-KO & \\
\hline & & & & & & $\begin{array}{l}\text { Session 5-8, } \\
\mathrm{F}_{1,38}=7.996 \\
p=0.0074\end{array}$ & \\
\hline \multirow{15}{*}{$\begin{array}{l}\text { Cued and } \\
\text { contextual fear } \\
\text { conditioning }\end{array}$} & $\begin{array}{l}\text { Fear conditioning } \\
\text { test }\end{array}$ & & & & & & \\
\hline & Conditioning & Freezing (\%) & --- & -- & $\begin{array}{l}F_{1,43}=32.949 \\
p<0.0001\end{array}$ & $\begin{array}{l}F_{1,38}=10.046 \\
p=0.003\end{array}$ & Fig. $8 \mathrm{a}, \mathrm{g}$ \\
\hline & $\begin{array}{l}\text { Context test } 1 \text { day } \\
\text { after conditioning }\end{array}$ & Freezing (\%) & --- & - & $\begin{array}{l}F_{1,43}=36.049 \\
p<0.0001\end{array}$ & $\begin{array}{l}F_{1,38}=6.837 \\
p=0.0127\end{array}$ & Fig. $8 b, h$ \\
\hline & $\begin{array}{l}\text { Cued test } 1 \text { day after } \\
\text { conditioning (pre-CS) }\end{array}$ & Freezing (\%) & - & -- & $\begin{array}{l}F_{1,43}=4.641 \\
p=0.0369\end{array}$ & $\begin{array}{l}F_{1,38}=10.947 \\
p=0.0021\end{array}$ & Fig. $8 b, h$ \\
\hline & $\begin{array}{l}\text { Cued test } 1 \text { day after } \\
\text { conditioning (CS) }\end{array}$ & Freezing (\%) & n.s. & - & $\begin{array}{l}F_{1,43}=4.003 \\
p=0.0517\end{array}$ & $\begin{array}{l}F_{1,38}=5.287 \\
p=0.0271\end{array}$ & Fig. $8 b, h$ \\
\hline & $\begin{array}{l}\text { Context test } 1 \text { month } \\
\text { after conditioning }\end{array}$ & Freezing (\%) & --- & - & $\begin{array}{l}F_{1,42}=36.559 \\
p<0.0001\end{array}$ & $\begin{array}{l}F_{1,38}=4.772 \\
p=0.0352\end{array}$ & Fig. $8 \mathrm{c}$, i \\
\hline & $\begin{array}{l}\text { Cued test } 1 \text { month after } \\
\text { conditioning (pre-CS) }\end{array}$ & Freezing (\%) & - & -- & $\begin{array}{l}F_{1,41}=6.521 \\
p=0.0145\end{array}$ & $\begin{array}{l}F_{1,38}=15.314 \\
p=0.0004\end{array}$ & Fig. $8 \mathrm{c}$, i \\
\hline & $\begin{array}{l}\text { Cued test } 1 \text { month } \\
\text { after conditioning (CS) }\end{array}$ & Freezing (\%) & - & - & $\begin{array}{l}\mathrm{F}_{1,41}=5.188 \\
p=0.028\end{array}$ & $\begin{array}{l}F_{1,38}=5.254 \\
p=0.0275\end{array}$ & Fig. $8 c, i$ \\
\hline & $\begin{array}{l}\text { Fear conditioning test } \\
\text { (foot shock 1) }\end{array}$ & $\begin{array}{l}\text { Distance } \\
\text { traveled }(\mathrm{cm})\end{array}$ & n.s. & ++ & $\begin{array}{l}F_{1,43}=10.489 \\
p=0.4883\end{array}$ & $\begin{array}{l}F_{1,38}=12.428 \\
p=0.0011\end{array}$ & Fig. 8d, j \\
\hline & $\begin{array}{l}\text { Fear conditioning test } \\
\text { (foot shock 2) }\end{array}$ & $\begin{array}{l}\text { Distance } \\
\text { traveled }(\mathrm{cm})\end{array}$ & n.s. & n.s. & $\begin{array}{l}F_{1,43}=0.423 \\
p=0.5189\end{array}$ & $\begin{array}{l}\mathrm{F}_{1,38}=0.347 \\
p=0.5592\end{array}$ & Fig. $8 d, j$ \\
\hline & $\begin{array}{l}\text { Fear conditioning test } \\
\text { (foot shock 3) }\end{array}$ & $\begin{array}{l}\text { Distance } \\
\text { traveled }(\mathrm{cm})\end{array}$ & n.s. & n.s. & $\begin{array}{l}F_{1,43}=1.026 \\
p=0.3168\end{array}$ & $\begin{array}{l}F_{1,38}=1.858 \\
p=0.1809\end{array}$ & Fig. $8 d, j$ \\
\hline & $\begin{array}{l}\text { Context test } 1 \text { day } \\
\text { after conditioning }\end{array}$ & $\begin{array}{l}\text { Activity suppression } \\
\text { ratio }\end{array}$ & ++ & n.s. & $\begin{array}{l}F_{1,43}=15.048 \\
p=0.0004\end{array}$ & $\begin{array}{l}F_{1,38}=1.699 \\
p=0.2002\end{array}$ & Fig. $8 \mathrm{e}, \mathrm{k}$ \\
\hline & $\begin{array}{l}\text { Context test } 1 \text { month } \\
\text { after conditioning }\end{array}$ & $\begin{array}{l}\text { Activity suppression } \\
\text { ratio }\end{array}$ & +++ & n.s. & $\begin{array}{l}F_{1,42}=46.445 \\
p<0.0001\end{array}$ & $\begin{array}{l}F_{1,38}=0.154 \\
p=0.697\end{array}$ & Fig. $8 \mathrm{e}, \mathrm{k}$ \\
\hline & $\begin{array}{l}\text { Cued test } 1 \text { day after } \\
\text { conditioning (CS) }\end{array}$ & $\begin{array}{l}\text { Activity suppression } \\
\text { ratio }\end{array}$ & n.s. & n.s. & $\begin{array}{l}F_{1,43}=0.211 \\
p=0.6482\end{array}$ & $\begin{array}{l}F_{1,38}=1.274 \\
p=0.2662\end{array}$ & Fig. 8f, I \\
\hline & $\begin{array}{l}\text { Cued test } 1 \text { month } \\
\text { after conditioning (CS) }\end{array}$ & $\begin{array}{l}\text { Activity suppression } \\
\text { ratio }\end{array}$ & n.s. & n.s. & $\begin{array}{l}F_{1,41}=3.032 \\
p=0.0891\end{array}$ & $\begin{array}{l}F_{1,38}=1.046 \\
p=0.3129\end{array}$ & Fig. 8f, I \\
\hline
\end{tabular}

n.s. no significance

Nominal significance: $+/-p<0.05,++/--p<0.01,+++/---p<0.001$

associated with increased locomotor activity and/or with impaired memory of Rapgef2-cKO mice as discussed later. However, in Rapgef6-KO mice, we failed to detect phenotypic alterations associated with depression using the tail suspension test (Fig. 3d). In the porsolt forced swim test, Rapgef6-KO mice exhibited decreased immobility that could be associated with their hyperlocomotion phenotypes (Fig. 3b). Reduction in anxiety-like behavior was detected in Rapgef2-cKO mice by the open field test, where the time spent in the arena center was significantly increased (Fig. 2b). Reduced anxiety-like behavior in Rapgef2-cKO mice was also evident in the elevated plus maze test, where the percentages of entries into the open arms and the time spent in the open arms were significantly increased (Fig. 2i, j), further supporting the notion obtained by the open field test. On the other hand, it might be hard to conclude that Rapgef6 deficiency affected anxiety-like behavior because the elevated plus maze test and the light/dark transition test failed to detect changes associated with the Rapgef6 genotype (Fig. 2g, h, k, l) although Rapgef6-KO mice exhibited mild changes in the time spent in the arena center in the early and late phases of the open field test (Fig. 2c, d).

To examine the effects on sociability, we performed social interaction tests (Fig. 5). Overall, social interaction tests with a novel environment (Fig. 5a-j) and those in a home cage (Fig. 5k-n) did not reveal significant genotype effects except for some indices. For the indices showing genotype dependency such as number of contacts (Fig. 5b, g) and total duration of active contacts (Fig. 5c, h), it was difficult to rule out the possibility that the increase associated with the genotypes 
was simply caused by the hyperlocomotion phenotypes solely based on the data obtained from these social interaction tests. However, Crawley's version of sociability tests demonstrated that Rapgef2-cKO mice preferred to stay around the stranger cage than control mice although the control animals that were compared to Rapgef2-cKO mice seemed slightly poor at discriminating an empty cage and a stranger-mouse-containing cage (Fig. 5o). These results indicated that the test detected increased sociability in Rapgef2-cKOmice (Fig. 5o). In contrast, such trends were not obviously detected with Rapgef6-KO mice (Fig. 5p). These results indicated that only Rapgef2 genotype affected sociability.

In the current study, our results demonstrated that Rapgef2-cKO mice and Rapgef6-KO mice exhibited defects in the learning and memory tasks. Data obtained by the T-maze test suggested impaired working memory in Rapgef2-cKO mice (Fig. 7a). Working memory defects were also detected in Rapgef6-KO mice even though the protocol employed was slightly different from that used for Rapgef2-cKO mice (Fig. 7b, c). Data obtained by the Barnes circular maze test suggested that the acquisition and retention of the spatial reference memory were impaired in Rapgef2-cKO mice (Fig. 6). On the other hand, Rapgef6-KO mice failed to show an obvious abnormality in this test, consistent with the previous report using the same Rapgef6 mutant strain [27]. Taken together, these results suggested that Rapgef2 deficiency, rather than Rapgef6 deficiency, decreases the learning ability. We also performed the contextual and cued fear conditioning test (Fig. 8). In this test, Rapgef2-cKO mice exhibited altered performance during the conditioning phase (Fig. 8a), which may reflect their reduced learning ability and/or hyperlocomotion phenotype. Their altered learning ability and hyperlocomotion phenotypes also seem to affect the outcomes of the tests performed on day 2 and 1 month after conditioning, where Rapgef2-cKO mice again exhibited reduced freezing in both context testing and cued testing with altered context (Fig. 8b, c). However, the activity suppression ratio indicates that Rapgef2 deficiency tended to reduce the performance more significantly 1 month after conditioning than on day 2 (Fig. 8e, f). Therefore, we cannot rule out the possibility that long-term memory maintenance might be impaired in Rapgef2-cKO mice. To clarify these points, further studies are required by employing another experimental system, such as that where Rapgef2 is inactivated after conditioning. In Rapgef6-KO mice, we also detected similar tendency, such as reduced freezing (Fig. 8g-i). However, the activity suppression ratio indicated that reduced freezing in Rapgef6-KO mice was caused as a result of their hyperlocomotive tendencies rather than by memory or learning defects (Fig. 8k, l). Contrary to our current results, previous studies performed at a different facility using the same Rapgef6-KO mouse strain concluded that Rapgef6-KO mice had deficiencies in context-dependent memory [27]. However, in that study, Levy et al., did not calculate activity suppression ratio and did not consider the hyperlocomotion phenotypes of Rapgef6-KO mice [27]. Therefore, we speculate that this discrepancy could be possibly attributed to the underestimation of the impact of the hyperlocomotion phenotypes on the outputs and/or that the differences in experimental facilities and experimenters. Impairment in learning and memory also seemed to affect the outputs of the porsolt forced swim test, in which both Rapgef2-cKO mice and Rapgef6-KO mice exhibited reduced immobility during the initial phases at day2 (Fig. 3a, b). Although their hyperlocomotion phenotypes could again affect the outputs of this test, another possibility is that Rapgef2-cKO mice and Rapgef6$\mathrm{KO}$ mice failed to memorize the previous fear experience due to the defects in learning and memory.

Rapgef2 and Rapgef6 were implicated in the etiology of schizophrenia by genome-wide association study (GWAS) of patients with the schizophrenic disorder [28, 29]. Therefore, we investigated whether the observed behavioral characteristics of Rapgef2-cKO and Rapgef6-KO mice were relevant to those of symptomatic schizophrenia patients. For instance, hyperlocomotion phenotypes in Rapgef2-cKO mice and Rapgef6-KO mice seemed to be analogous to psychomotor agitation evident in schizophrenia patients [46]. Additionally, mice models with psychomotor agitation-like phenotypes, such as those produced by psychostimulant administration, are recognized as a model for schizophrenia $[47,48]$. T-maze alternation memory task and Barnes maze indicated a working memory defect and a spatial memory defect, respectively, in Rapgef2-cKO mice and Rapgef6-KO mice. These defects were reported to be a phenotype of schizophrenia-relevant behavior [47]. However, as discussed above, Rapgef2-cKO mice exhibited increased sociability, which seems opposite to the widely accepted concept that reduced sociability recapitulated in experimental animals is a model of social withdrawal observed as a negative symptom of schizophrenia. These suggest that behavioral abnormalities in Rapgef2-cKO mice and Rapgef6-KO mice recapitulate not only those of a subpopulation of schizophrenia but also those seen in other mental disorder patients.

Rap1 and Rap2 were reported to be involved in the regulation of higher brain functions, such as fear learning and spatial learning $[10,12]$. Previous studies focused on Rap proteins as molecules coupling cAMP signaling to the signaling involving extracellular signal regulated kinase (ERK) 1 and ERK2, which regulates excitability, synaptic plasticity, learning and memory [49]. In such cAMP-dependent Rap activation, Rapgef3 and Rapgef4 (also known as Epac1 and Epac2, respectively) have been biochemically characterized as cAMP- 
dependent Rap-specific GEFs and shown to be involved in spatial learning and social interaction [16, 50, 51]. In contrast to these 2 cAMP-responsive Rap GEFs, Rapgef2 and Rapgef6 cannot be activated by cAMP as we and others previously reported [18-20,22], and thus they have a role other than transmitting cAMP signaling to Rap activation. In this regard, our study expands our knowledge on the Rap signaling in the regulation of higher brain functions.

To the best of our knowledge, this is the first report that demonstrates that Rapgef2 is involved in regulation of locomotion and working memory. Further, we observed that Rapgef2 deficiency produced more pronounced defects in the higher brain functions although both Rapgef2 and Rapgef6 share structural and functional similarities. Considering that Rapgef2 and Rapgef6 are abundantly expressed in the cortex $[26,27]$, they may play an important role in prefrontal cortex-dependent working memory. Further studies at the cellular and molecular levels are required to clarify the mechanisms of Rapgef2- and/or Rapgef6-mediated regulation of the activities of neurons, which may help in understanding the role of Rapgef 2 and Rapgef6 in the regulation of higher brain functions and the impact of their deficiencies in the development of mental disorders.

\section{Methods}

\section{Animals and experimental design}

Mice used in this study had been backcrossed over 11 times to the C57BL/6 J strain. Rapgef2-cKO (Rapgef2 ${ }^{\text {flox/lox; }}$; $\left.E m x I^{\text {cre/+}}\right)$ mice were prepared by mating Rapgef $2^{\text {flox/flox }}$ mice with $E m x^{\mathrm{Cre} /+}$ mice [24]. Rapgef6-KO mice were generated as detailed previously [35]. Behavioral tests were performed with male mice that were at least 14 weeks old. The ages of the mice at the time of each experiment are listed in Tables 2 and 3. In each strain, we run the experiments with a single batch. Mice were group housed in a room with a $12 \mathrm{~h}$ light/dark cycle (lights on at 7:00 am) with free access to food and water expect for the period during which the T-maze test was being conducted. Room temperature was kept at $23 \pm 2{ }^{\circ} \mathrm{C}$. Behavioral testing was performed between 9:00 am and 6:00 pm. After tests, all apparatus used was cleaned with diluted hypochlorite solution or $70 \%$ ethanol to prevent a bias from olfactory cues. To minimize the effects of previous tests on the subsequent tests, we performed the behavioral test battery in the following order: general health and neurological screens, light/dark transition test, open field test, elevated plus maze, hot plate test, social interaction test in a novel environment, rotarod test, Crawley's sociability and preference for social novelty test, startle response/ prepulse inhibition test, Porsolt forced swim test, Barnes maze test, T-maze test, tail suspension test, fear conditioning test, and social interaction test in a home
Table 2 Comprehensive behavioral test battery of Rapgef2-cKO mice

\begin{tabular}{|c|c|}
\hline Test & Age (w) \\
\hline 1. General health & $17-23$ \\
\hline 2. Light/dark transition & $17-24$ \\
\hline 3. Open field & $18-24$ \\
\hline 4. Elevated plus-maze & $18-24$ \\
\hline 5. Hot plate & $19-25$ \\
\hline 6. Social Interaction (novel environment) & $19-25$ \\
\hline 7. Rotarod & $19-26$ \\
\hline 8. Social Interaction (Crawley version) & $22-29$ \\
\hline 9. Prepulse inhibition & $23-30$ \\
\hline 10. Porsolt forced swim & $24-30$ \\
\hline 11. Barnes maze (training) & $42-52$ \\
\hline 12. Barnes maze (probe test (24 h)) & $46-52$ \\
\hline 13. Barnes maze (probe test (1 month)) & $51-57$ \\
\hline 14. T-maze (forced alternation with fasting) & $60-66$ \\
\hline 15. T-maze (left-right discrimination) & $61-69$ \\
\hline 16. Tail suspension test & $63-69$ \\
\hline 17. Fear conditioning test (Day 1) & $63-70$ \\
\hline 18. Fear conditioning test (Day 2) & $64-70$ \\
\hline 19. Fear conditioning test (Day 31) & $68-74$ \\
\hline 20. Social Interaction (home cage) & $69-76$ \\
\hline 21. Open field & $72-78$ \\
\hline
\end{tabular}

Age $(w)$ age in weeks of mice at the beginning of each test listed

cage. Behavioral tests were performed at intervals of at least 1 day. The use and care of the animals were reviewed and approved by the Institutional Animal Care and Use Committee of Kobe University and that of Fujita Health University. Raw data of the behavioral tests are available at the mouse phenotype database (http://www.mouse-phenotype.org).

\section{Behavioral tests \\ General health and neurological screens}

The presence of whiskers or bald hair patches was recorded. The righting, whisker touch, and ear twitch reflexes were also evaluated. Body weight and rectal temperature were measured. Neuromuscular strength was tested with the grip strength test and wire hang test. Grip strength was measured by a grip strength meter (O'HARA \& Co., Tokyo, Japan). Mice were grasped a wire grid by the forelimbs and pulled backward until they release it. The peak force was recorded in Newtons (N). Each mouse was tested three times, and the greatest value obtained was used for further data analyses. In the wire hang test, a mouse was placed on the wire mesh at the top of apparatus (O'HARA \& Co., Tokyo, Japan), and the wire mesh was then gently turned inside out. The mouse gripped the 
Table 3 Comprehensive behavioral test battery of Rapgef6-KO mice

\begin{tabular}{ll}
\hline Test & Age (w) \\
\hline 1. General health & $14-16$ \\
2. Rotarod & $20-22$ \\
3. Hot plate & $18-19$ \\
4. Open field & $15-17$ \\
5. Light/dark transition & $15-16$ \\
6. Elevated plus-maze & $17-19$ \\
7. Porsolt forced swim & $32-34$ \\
8. Tail suspension test & $41-42$ \\
9. Prepulse inhibition & $32-33$ \\
10. Social Interaction (novel environment) & $19-20$ \\
11. Social Interaction (home cage) & $59-62$ \\
12. Social Interaction (Crawley version) & $31-33$ \\
13. Barnes maze (training) & $32-36$ \\
14. Barnes maze (probe test (24 h)) & $34-36$ \\
15. Barnes maze (probe test (1 month)) & $39-40$ \\
16. T-maze (forced alternation without fasting) & $39-42$ \\
17. Fear conditioning test (Day 1) & $41-43$ \\
18. Fear conditioning test (Day 2) & $41-43$ \\
19. Fear conditioning test (Day 36) & $46-48$ \\
\hline Age (w) age in weeks of mice at the beginng of each test listed
\end{tabular}

Age (w) age in weeks of mice at the beginning of each test listed

wire in order not to fall off, and the latency to fall was recorded with 60-s cut-off time. A one-way ANOVA was used to determine $p$ values for genotype effects.

\section{Rotarod test}

An accelerating rotarod (UGO Basile, Comerio, VA, Italy) was used to test the motor coordination and balance of mice. A mouse was placed on a rotating drum $(3 \mathrm{~cm}$ diameter) and the speed of the rotarod was accelerated from 4 to $40 \mathrm{rpm}$ in $5 \mathrm{~min}$. The animals went through three trials in a day on 2 consecutive days. The length of the period that a mouse was able to maintain its balance on the rod was determined. A two-way repeated measures ANOVA was used to determine $p$ values for genotype effects.

\section{Hot plate test}

To evaluate the sensitivity to painful stimuli, a mouse was placed on a hot plate (Columbus Instruments, $\mathrm{OH}, \mathrm{USA}$ ) at $55.0( \pm 0.1)^{\circ} \mathrm{C}$. The latency to the first fore-paw or hindpaw response, defined by either a foot shake or a paw lick, was recorded with a $15 \mathrm{~s}$ cut-off time. A one-way ANOVA was used to determine $p$ value for genotype effect.

\section{Open field test}

Locomotor activity was measured using an open field apparatus $(40 \times 40 \times 30 \mathrm{~cm}$; Accuscan Instruments, Columbus, $\mathrm{OH}, \mathrm{USA}$ ) [52]. The test chamber was illuminated at $100 \pm 5 \mathrm{~lx}$. A mouse was placed in the corner of the apparatus. Total distance traveled $(\mathrm{cm})$ and time spent in the center area of the open field $(20 \times 20 \mathrm{~cm})$ were recorded by the VersaMax system. Data were collected for $120 \mathrm{~min}$. A two-way repeated measures ANOVA was used to determine $p$ values for genotype effects.

\section{Light/dark transition test}

The light/dark transition test was performed as previously described $[52,53]$. The apparatus comprised a box $(21 \times 42 \times 25 \mathrm{~cm})$ that was divided equally into $2 \mathrm{sec}-$ tions with a partition having a door (O'HARA \& Co., Tokyo, Japan). One chamber was brightly illuminated $(390 \pm 5 \mathrm{~lx})$ whereas the other one was kept dark (2 lx). A mouse was placed into the dark chamber and allowed to move freely between the 2 chambers through the door open for $10 \mathrm{~min}$. Total number of transitions and latency to the first enter to the light chamber were recorded using ImageLD software. A one-way ANOVA was used to determine $p$ values for genotype effects.

\section{Elevated plus-maze test}

To evaluate anxiety-related behavior, the elevated plus maze test was conducted as previously described [52, 54]. The apparatus consisted of 2 open arms $(25 \times 5 \mathrm{~cm})$ and 2 arms enclosed with $15 \mathrm{~cm}$ high transparent walls, all of which were elevated $55 \mathrm{~cm}$ above the floor (O'HARA \& Co., Tokyo, Japan). Arms of the same type were located opposite from each other. In order to minimize the chance that animals fell from the apparatus, 3-mm high plexiglas ledges were equipped in the open arms. A mouse was placed in the central square of the maze $(5 \times 5 \mathrm{~cm})$ so that it faced one of the enclosed arms. Number of entries into the each kind of arms and time spent (sec) in each kind of arms were recorded for $10 \mathrm{~min}$. Percentage of entries into the open arms and that of time spent in the open arms were determined. Data acquisition and analysis were performed automatically using ImageEP software. A one-way ANOVA was used to determine $p$ values for genotype effects.

\section{Porsolt forced swim test}

A transparent plastic cylinder $(22 \mathrm{~cm}$ height $\times 12 \mathrm{~cm}$ diameter), which was filled with water (approximately $23{ }^{\circ} \mathrm{C}$ ) up to a height of $7.5 \mathrm{~cm}$, was put in a white plastic chamber (O'HARA \& Co., Tokyo, Japan). A mouse was placed in the cylinders, and the immobility and the distance traveled were recorded for $10 \mathrm{~min}$ by capturing images at 2 frames per second on days 1 and 2 [52]. For each pair of successive frames, the amount of area (pixels) in which the mouse moved was measured. If the amount of area was below a certain threshold, which was optimized by human observation of the behavior, the behavior was classified as "immobile". Immobility 
lasting for less than $2 \mathrm{~s}$ was not included in the analyses. Data acquisition and analysis were performed automatically using ImageTS software. A two-way repeated measures ANOVA was used to determine $p$ values for genotype effects. In addition, $p$ values for genotype $\times$ time interaction $(g \times t)$ were also determined.

\section{Tail suspension test}

Mice were suspended with their tail by adhesive tape at $30 \mathrm{~cm}$ above the floor of a white plastic chamber (O'HARA \& Co., Tokyo, Japan), and their behavior was recorded for $10 \mathrm{~min}$ [53]. Similar to the Porsolt forced swim test, images were captured at 2 frames per second, and immobility was judged by the application program according to a certain threshold, which was optimized by human observation of the behavior. Data acquisition and analysis were performed automatically using ImageTS software. A two-way repeated measures ANOVA was used to determine $p$ values for genotype effects. Also, $p$ values for genotype $\times$ time interaction $(g \times t)$ were determined.

\section{Startle response/Prepulse inhibition test}

A startle reflex measurement system was used (O'HARA \& Co., Tokyo, Japan) [49]. A test session began by placing a mouse in a plastic cylinder where it was left undisturbed for $10 \mathrm{~min}$. White noise $(40 \mathrm{msec})$ was used as the startle stimulus for all trial types. The startle response was measured for $400 \mathrm{msec}$ by accelerometer from $100 \mathrm{msec}$ before the onset of the prepulse stimulus. The background noise level in each chamber was $70 \mathrm{~dB}$. A test session consisted of 2 types for startle stimulusonly trials (110 or $120 \mathrm{~dB}$ ) and 4 types for prepulse inhibition trials (74-110, 78-110, 74-120, and 78-120 dB). The prepulse sound was presented $100 \mathrm{msec}$ before startle stimulus. Six trial types were presented in a pseudorandom order, such that each trial type was presented once within a block. The average inter-trial interval was $15 \mathrm{~s}$ (range: 10-20 s). A two-way repeated measures ANOVA was used to determine $p$ values for genotype effects.

\section{Social interaction test in a novel environment}

Two mice of identical genotype that had been housed in different cages were placed together in a box $(40 \times 40 \times$ $30 \mathrm{~cm}$ ) (O'HARA \& Co., Tokyo, Japan), and allowed to explore freely for $10 \mathrm{~min}$ the box [52]. Total duration of contacts, total number of contacts, total duration of active contacts, mean duration of a contact, and total distance traveled were measured. If the 2 mice contacted each other and the distance traveled by either mouse was longer than $10 \mathrm{~cm}$, the behavior was considered as an 'active contact'. Behavior was recorded and analyzed automatically using ImageSI program. Images were captured at 3 frames per second. A one-way ANOVA was used to determine $p$ values for genotype effects.

\section{Social interaction test in a home cage}

The system for monitoring social interaction comprised a home cage and a filtered cage top with an infrared video camera $(31 \times 19 \times 30 \mathrm{~cm} ; 25 \times 15 \times 23.5 \mathrm{~cm}$, inside dimensions) (O'HARA \& Co., Tokyo, Japan) [55]. Two mice with the same genotype that had been housed separately were placed together in a home cage. To evaluate social interaction, their social behavior was monitored with a video camera for a week. The occurrence of social interaction was detected by counting the number of particles consisting of the mice as follows: 2 particles indicated that the mice were not in contact whereas 1 particle indicated that 2 mice were in contact. Locomotor activity of the mice was also measured by quantifying the number of pixels that changed between each pair of successive frames while these experiments were performed. Analysis was automatically performed using ImageHA software. A twoway repeated measures ANOVA was used to determine $p$ values for genotype effects.

\section{Crawley's sociability and social novelty preference test}

To investigate the effect of complex genetics on sociability and preference for social novelty, Crawley's sociability and social novelty preference test was performed as described $[36,37]$ using an apparatus composed of a threechambered rectangular box with an infrared video camera (O'HARA \& Co., Tokyo, Japan). Each chamber $(20 \times 40 \times$ $47 \mathrm{~cm}$ ) was partitioned by a wall with a small square opening $(5 \times 3 \mathrm{~cm})$ thus allowing mice to access each area. Wire cages $(11 \mathrm{~cm}$ height $\times 9 \mathrm{~cm}$ diameter, vertical bars 0 . $5 \mathrm{~cm}$ apart), which allowed nose contact between the bars but prevented fighting, were located in the corners of side chambers. "Habituation" session was performed in the apparatus for $10 \mathrm{~min}$. In "sociability" test, an unfamiliar C57BL/6 J male (Stranger 1) was enclosed in the wire cage. The cage containing Stranger 1 was placed in one of the side chambers while the cage placed in the other side chamber was kept empty. A subject mouse was placed in the middle chamber and allowed to move freely for $10 \mathrm{~min}$. To perform "preference" test, after completion of the first 10-min session, a second unfamiliar mouse (Stranger 2) was enclosed in the other wire cage, which had been empty in the first session, and the subject mouse was again placed in the middle chamber and examined as in the first session. In each session, the amount of time spent around each cage was measured. Data acquisition and analysis were performed using ImageCSI. A twoway repeated measures ANOVA was used to determine $p$ values for genotype effects whereas paired $t$-test was employed to derive $p$ values between the types of the apparatuses. To examine the effects of Rapgef2 deficiency, after the "habituation" was completed for all individuals to be tested by the following tests, each individual was subjected to the "sociability" test and the "preference" test 
sequentially. To examine the effects of Rapgef6 deficiency, each individual was subjected to the "habituation" and then subjected to the "sociability" test and the "preference" test sequentially.

\section{Barnes maze test}

To assess spatial learning, Barnes circular maze was used. The test was conducted on "dry land", a white circular surface with a diameter of $1.0 \mathrm{~m}$ having 12 holes equally spaced around the perimeter (O'HARA \& Co., Tokyo, Japan). A black Plexiglas escape box $(17 \times 13 \times 7 \mathrm{~cm})$, which had paper bedding on its bottom, was located under one of the holes. The hole above the escape box represented the target. The spatial location of the target was consistent for a given mouse but randomized across mice subjected to the tests. The maze was rotated daily to prevent a bias caused by olfactory or proximal cues in the maze. The spatial location of the target was unchanged with respect to the visual room cues. During acquisition test, each trial ended when a mouse entered the escape box or after $5 \mathrm{~min}$ had elapsed. Mice that failed to find the box were guided to it. Latency and distance to reach the target hole, number of errors (defined by placing mouse's nose in a hole that did not have an escape box) were recorded using ImageBM software. A one-way ANOVA and two-way repeated measures ANOVA was used to determine $p$ values for genotype effects. One day after the last training, a probe trial was performed using the same apparatus, from which the escape box was omitted, to assess the memory based on distal environmental cues. Another probe test was performed 1 month later to evaluate retention of spatial memory. In the probe tests, time spent around the target hole was also recorded by ImageBM software. To judge whether the differences observed were statistically significant, $p$ values for genotype effects determined by a one-way ANOVA. For the time spent around each hole, $p$ values for genotype effects and those for target $\times$ genotype interaction were determined by a two-way repeated measures ANOVA. For the time spent around the target and its adjacent holes, $p$ values for genotype effects and for target $\times$ genotype interaction were determined by a two-way repeated measures ANOVA, and those between the target and its adjacent holes for each genotype were determined by paired $t$-test.

\section{T-maze test}

To assess working memory, we employed a forced alternation task test with food deprivation (for examining the effects of Rapgef2 deficiency) and a forced alternation task test without food deprivation (for examining the effects of Rapgef6 deficiency) using T-maze apparatus (O'HARA \& Co., Tokyo, Japan) [55]. To assess the effects of Rapgef2 deficiency, mice were subjected to the forced alternation task test for 4 days (one session consisting of 10 trials per day; cut-off time, $50 \mathrm{~min}$ ). In this forced alternation test, before the pre-training, mice to be subjected to the test were deprived of food until their bodyweight was reduced to $80-85 \%$ of their initial bodyweight. On the other hand, to assess the effects of Rapgef6 deficiency, mice were tested with another protocol for the forced alternation task test from which fasting before the test was omitted. Each trial consisted of first and second runs. On the first run of each trial, a mouse was forced to choose one of the arms of the $\mathrm{T}$ (area A1 or A2). After the mouse stayed more than $10 \mathrm{~s}$, sliding doors that separated the arms (areas A1 andA2) and the connecting passage ways (areas P1 andP2) opened so that the mouse could return to the starting compartment (area S1) through the connecting passage ways by itself. The mouse was then given a $3 \mathrm{~s}$ delay there, followed by a free choice between the arms of the $\mathrm{T}$ arm. The correct answer was the other arm that had not been chosen on the first run. On day 5-8, a delay (3, $60,120,180 \mathrm{~s})$ was applied after the first run. Data acquisition, control of sliding doors, and data analysis were performed by ImageTM software. A two-way repeated measures ANOVA was used to determine $p$ value for genotype effects was determined.

\section{Contextual and cued fear conditioning test}

A contextual and cued fear conditioning test was conducted as previously described [52, 56]. To assess fear related learning and memory, each mouse was placed in a test chamber $(33 \times 25 \times 28 \mathrm{~cm})$ with a stainless-steel grid floor $(0.2 \mathrm{~cm}$ diameter, spaced $0.5 \mathrm{~cm}$ apart $)$ (O'HARA \& Co., Tokyo, Japan) illuminated at $100 \pm 5 \mathrm{~lx}$ and allowed to explore freely for $2 \mathrm{~min}$. Conditioned stimulus (55 dB white noise) was presented for $30 \mathrm{~s}$, followed by a mild foot shock $(2 \mathrm{~s}, 0.3 \mathrm{~mA})$, which served as the unconditioned stimulus (US). Two more CS-US pairings were presented with a 2-min inter-stimulus interval. Context testing was conducted 1 day and 1 month after conditioning in the same chamber for $300 \mathrm{~s}$ to each mouse. Cued test with an altered context was conducted 1 day and 1 month after conditioning using a triangular box $(33 \times 29 \times 32 \mathrm{~cm})$ (O'HARA \& Co., Tokyo, Japan), which was located in a different room. The test chamber was illuminated at $30 \pm 5 \mathrm{~lx}$. Tone stimulus for the cued test was applied for 180 s. During the test, images were captured at 1 frame per second. For each pair of successive frames, the amount of area (pixels) by which the mouse moved was measured. When this area was below a certain threshold (i.e., 30 pixels), the behavior was classified as "freezing". The optimal threshold (amount of pixels) used to classify freezing was determined by adjusting it to the amount of freezing measured by human observation. "Freezing" that lasted less than the defined time threshold (i.e., $2 \mathrm{~s}$ ) was not included in the analysis. Data acquisition 
and analysis were performed with Image FZ. Activity suppression ratio was calculated as follows: suppression ratio $=$ (activity during testing $) /$ (activity during baseline + activity during testing) [56]. To judge whether the differences observed were statistically significant, $p$ values for genotype effects were determined either by a two-way repeated measures ANOVA (excepting for activity suppression ratio) or by a one-way ANOVA (for activity suppression ratio).

\section{Data analysis}

All behavioral data were automatically collected using application softwares, which were derivatives of Image J program optimized for each type of tests by Tsuyoshi Miyakawa (available through O'HARA \& Co., Tokyo, Japan). For statistical analysis, StatView (SAS Institute, Cary, NC, USA) was used. Methods for statistical analysis, including a paired $t$-test, one-way ANOVAs, and two-way repeated measures ANOVAs, are described above. Data are represented as mean \pm standard error of the mean (SEM). If a $p$ value was smaller than 0.05 , the difference was considered statistically significant.

\section{Abbreviations}

CC: Corpus callosum; cKO: Conditional knockout; ECM: Ectopic cortical mass; GEF: Guanine nucleotide exchange factor; GHNS: General health and neurological screens; GWAS: Genome-wide association study; KO: Knockout; RGC: Radial glial cell

\section{Acknowledgments}

We thank Dr. T. Terashima, Dr. T. Setsu and Mr. Y. Sakihama at Kobe University Graduate School of Medicine for their helpful comments and discussions. We also thank members of Dr. Kataoka's laboratory.

\section{Funding}

This research was supported by Grant-in-Aid for Scientific Research on Innovative Areas (Comprehensive Brain Science Network and Platform of Advanced Animal Model Support) from the Ministry of Education, Culture, Sports, Science and Technology (MEXT) of Japan. Behavioral analysis was carried out at Institute for Comprehensive Medical Science, Fujita Health University (Joint Usage/Research Center for Genes, Brain and Behavior accredited by MEXT) in Japan. These funding agencies did not participate in designing the study, collecting, analyzing, or interpreting data, or writing the manuscript.

\section{Availability of data and materials}

Raw data of the behavioral tests of the current study are available at the mouse phenotype database (http://www.mouse-phenotype.org).

\section{Authors' contributions}

TK and TM were responsible for the original concept and the overall design of the research. SB, KM and JI prepared the mice for behavioral tests. SH and JI performed the behavioral tests and analyzed the data. KM, SH, HE, TM and TK wrote the manuscript. All authors read and approved the final manuscript.

\section{Ethics approval and consent to participate}

This study does not involve human participants, human data or human tissues. Animal experiments were approved by the Animal Care and Use Committee of Kobe University and by the Animal Care and Use Committee of Fujita Health University.

\section{Competing interests}

The authors declare that they have no competing interests.

\section{Publisher's Note}

Springer Nature remains neutral with regard to jurisdictional claims in published maps and institutional affiliations.

\section{Author details}

${ }^{1}$ Division of Molecular Biology, Department of Biochemistry and Molecular Biology, Kobe University Graduate School of Medicine, 7-5-1 Kusunoki-cho, Chuo-ku, Kobe 650-0017, Japan. ${ }^{2}$ Division of Systems Medical Science, Institute for Comprehensive Medical Science, Fujita Health University, 1-98 Dengakugakubo, Kutsukake-cho, Toyoake, Aichi 470-1192, Japan. ${ }^{3}$ Present address: Department of Neurotherapeutics, Osaka University Graduate School of Medicine, 2-2Yamadaoka, Suita, Osaka 565-0871, Japan. ${ }^{4}$ Present address: Department of Biochemistry, Faculty of Pharmacy, Suez Canal University, El-shikh Zayed, Ismailia 41522, Egypt.

Received: 1 February 2018 Accepted: 29 April 2018

Published online: 10 May 2018

\section{References}

1. Gloerich M, Bos JL. Regulating Rap small G-proteins in time and space. Trends Cell Biol. 2011;21:615-23.

2. Knox AL, Brown NH. Rap1 GTPase regulation of adherens junction positioning and celladhesion. Science. 2002;295:1285-8.

3. Franco SJ, Martinez-Garay I, Gil-Sanz C, Harkins-Perry SR, Müller U. Reelin regulatescadherin function via Dab1/Rap1 to control neuronal migration and lamination in theneocortex. Neuron. 2011;69:482-97.

4. Jossin Y, Reelin CJA. Rap1 and N-cadherin orient the migration of multipolar neurons in the developing neocortex. Nat Neurosci. 2011;14:697-703.

5. Shah B, Lutter D, Tsytsyura Y, Glyvuk N, Sakakibara A, Klingauf J, et al. Rap1 GTPases are master regulators of neural cell polarity in the developing neocortex. Cereb Cortex. 2017;27:1253-69.

6. Zhu JJ, Qin Y, Zhao M, Van Aelst L, Ras MR. Rap control AMPA receptor trafficking during synaptic plasticity. Cell. 2002;1 10:443-55.

7. Zhu Y, Pak D, Qin Y, McCormack SG, Kim MJ, Baumgart JP, et al. Rap2-JNK removes synaptic AMPA receptors during depotentiation. Neuron. 2005;46:905-16.

8. Fu Z, Lee SH, Simonetta A, Hansen J, Sheng M, Pak DT. Differential roles of Rap1 and Rap2 small GTPases in neurite retraction and synapse elimination in hippocampal spiny neurons. J Neurochem. 2007;100:118-31.

9. Huang CC, You JL, Wu MY, Hsu KS. Rap1-induced p38 mitogen-activated protein kinase activation facilitates AMPA receptor trafficking via the GDI. Rab5 complex. J Biol Chem. 2004;279:12286-92.

10. Pan BX, Vautier F, Ito W, Bolshakov VY, Morozov A. Enhanced cortico-amygdala efficacy and suppressed fear in absence of Rap1. J Neurosci. 2008;28:2089-98,

11. Subramanian J, Dye L, Morozov A. Rap1 signaling prevents L-type calcium channel-dependent neurotransmitter release. J Neurosci. 2013;33:7245-52.

12. Ryu J, Futai K, Feliu M, Weinberg R, Sheng M. Constitutively active Rap2 transgenic mice display fewer dendritic spines, reduced extracellular signalregulated kinase signaling, enhanced long-term depression, and impaired spatial learning and fear extinction. J Neurosci. 2008;28:8178-88.

13. Bos JL, Rehmann H, Wittinghofer A. GEFs and GAPs: critical elements in the control of small G proteins. Cell. 2007;129:865-77.

14. Woolfrey KM, Srivastava DP, Photowala H, Yamashita M, Barbolina MV, Cahill $M E$, et al. Epac2 induces synapse remodeling and depression and its disease-associated forms alter spines. Nat Neurosci. 2009;12:1275-84.

15. Srivastava DP, Woolfrey KM, Jones KA, Anderson CT, Smith KR, Russell TA, et al. An autism-associated variant of Epac2 reveals a role for Ras/Epac2 signaling in controlling basal dendrite maintenance in mice. PLoS Biol. 2012;10:e1001350.

16. Yang $Y$, Shu $X$, Liu $D$, Shang $Y$, Wu Y, Pei L, et al. EPAC null mutation impairs learning and social interactions via aberrant regulation of miR-124 and Zif268 translation. Neuron. 2012;73:774-88.

17. Ballif BA, Arnaud L, Arthur WT, Guris D, Imamoto A, Cooper JA. Activation of a Dab1/CrkL/C3G/Rap1 pathway in Reelin-stimulated neurons. Curr Biol. 2004; 14:606-10

18. de Rooij J, Boenink NM, van Triest M, Cool RH, Wittinghofer A, Bos JL. PDZGEF1, a guanine nucleotide exchange factor specific for Rap1 and Rap2. J Biol Chem. 1999;274:38125-30.

19. Liao Y, Kariya K, Hu CD, Shibatohge M, Goshima M, Okada T, et al. RA-GEF, a novel Rap1A guanine nucleotide exchange factor containing a Ras/Rap1Aassociating domain, is conserved between nematode and humans. J Biol Chem. 1999;274:37815-20. 
20. Ohtsuka T, Hata Y, Ide N, Yasuda T, Inoue E, Inoue T, et al. nRap GEP: a novel neural GDP/GTP exchange protein for rap1 small G protein that interacts with synaptic scaffolding molecule (S-SCAM). Biochem Biophys Res Commun. 1999:265:38-44.

21. Pham N, Cheglakov I, Koch CA, de Hoog CL, Moran MF, Rotin D. The guanine nucleotide exchange factor CNrasGEF activates ras in response to CAMP and CGMP. Curr Biol. 2000;10:555-8.

22. Gao X, Satoh T, Liao Y, Song C, Hu CD, Kariya Ki K, et al. Identification and characterization of RA-GEF-2, a Rap guanine nucleotide exchange factor that serves as a downstream target of M-Ras. J Biol Chem. 2001;276:42219-25.

23. Kanemura H, Satoh T, Bilasy SE, Ueda S, Hirashima M, Kataoka T. Impaired vascular development in the yolk sac and allantois in mice lacking RA-GEF1. Biochem Biophys Res Commun. 2009;387:754-9.

24. Bilasy SE, Satoh T, Ueda S, Wei P, Kanemura H, Aiba A, et al. Dorsal telencephalon-specific RA-GEF-1 knockout mice develop heterotopic cortical mass and commissural fiber defect. Eur J Neurosci. 2009;29:1994-2008.

25. Bilasy SE, Satoh T, Terashima T, Kataoka T. RA-GEF-1 (Rapgef2) is essential for proper development of the midline commissures. Neurosci Res. 2011;71:200-9.

26. Maeta K, Edamatsu H, Nishihara K, Ikutomo J, Bilasy SE, Kataoka T. Crucia role of Rapgef2 and Rapgef6, a family of guanine nucleotide exchange factors for Rap1 small GTPase, in formation of apical surface adherens junctions and neural progenitor development in the mouse cerebral cortex. eNeuro. 2016;3 https://doi.org/10.1523/ENEURO.0142-16.2016.

27. Levy RJ, Kvajo M, Li Y, Tsvetkov E, Dong W, Yoshikawa Y, et al. Deletion of Rapgef6, a candidate schizophrenia susceptibility gene, disrupts amygdala function in mice. Transl Psychiatry. 2015;5:e577.

28. Xu B, Roos JL, Levy S, van Rensburg EJ, Gogos JA, Karayiorgou M. Strong association of de novo copy number mutations with sporadic schizophrenia. Nat Genet. 2008;40:880-5.

29. Xu B, Woodroffe A, Rodriguez-Murillo L, Roos JL, van Rensburg EJ, Abecasis $G R$, et al. Elucidating the genetic architecture of familial schizophrenia using rare copy number variant and linkage scans. Proc Natl Acad Sci U S A. 2009; 106:16746-51.

30. Barkovich AJ, Kuzniecky RI. Gray matter heterotopia. Neurology. 2000;55: 1603-8.

31. Pletnikov MV, Ayhan Y, Nikolskaia O, Xu Y, Ovanesov MV, Huang H, et al. Inducible expression of mutant human DISC1 in mice is associated with brain and behavioral abnormalities reminiscent of schizophrenia. Mol Psychiatry. 2008;13:173-86.

32. Hikida T, Jaaro-Peled H, Seshadri S, Oishi K, Hookway C, Kong S, et al. Dominant-negative DISC1 transgenic mice display schizophrenia-associated phenotypes detected by measures translatable to humans. Proc Natl Acad Sci U S A. 2007;104:14501-6.

33. Shen S, Lang B, Nakamoto C, Zhang F, Pu J, Kuan SL, et al. Schizophreniarelated neural and behavioral phenotypes in transgenic mice expressing truncated Disc1. J Neurosci. 2008;28:10893-904.

34. Jaaro-Peled H, Ayhan Y, Pletnikov MV, Sawa A. Review of pathological hallmarks of schizophrenia: comparison of genetic models with patients and nongenetic models. Schizophr Bull. 2010;36:301-13.

35. Yoshikawa Y, Satoh T, Tamura T, Wei P, Bilasy SE, Edamatsu H, et al. The M-RasRA-GEF-2-Rap1 pathway mediates tumor necrosis factor-alpha dependent regulation of integrin activation in splenocytes. Mol Biol Cell. 2007;18:2949-59.

36. Crawley JN. Designing mouse behavioral task relevant to autistic-like behaviors. Ment Retard Dev Disabil Res Rev. 2004;10:248-58.

37. Moy SS, Nadler JJ, Perez A, Barbaro RP, Johns JM, Magnuson TR, et al. Sociability and preference for social novelty in five inbred strains: an approach to assess autistic-like behavior in mice. Gens Brain Behav. 2004;3:287-302.

38. Anagnostaras SG, Josselyn SA, Frankland PW, Silva AJ. Computer-assisted behavioral assessment of Pavlovian fear conditioning in mice. Learn Mem. 2000;7:58-72.

39. Frankland PW, Bontempi B, Talton LE, Kaczmarek L, Silva AJ. The involvement of the anterior cingulate cortex in remote contextual fear memory. Science. 2004;304:881-3.

40. Squire LR, Wixted JT. The cognitive neuroscience of human memory since H.M. Annu Rev Neurosci. 2011;34:259-88.

41. Mihrshahi R. The corpus callosum as an evolutionary innovation. J Exp Zool B Mol Dev Evol. 2006;306:8-17.

42. Olton DS, Walker JA, Wolf WA. A disconnection analysis of hippocampal function. Brain Res. 1982;233:241-53.
43. MacPherson P, McGaffigan R, Wahlsten D, Nguyen PV. Impaired fear memory, altered object memory and modified hippocampal synaptic plasticity in split-brain mice. Brain Res. 2008;1210:179-88.

44. Ribeiro AS, Eales BA, Biddle FG. Short-term and long-term memory deficits in handedness learning in mice with absent corpus callosum and reduced hippocampal commissure. Behav Brain Res. 2013;245:145-51.

45. Sengar AS, Ellegood J, Yiu AP, Wang H, Wang W, Juneja SC, et al. Vertebrate intersectin 1 is repurposed to facilitate cortical midline connectivity and higher order cognition. J Neurosci. 2013;33:4055-65.

46. Gainetdinov RR, Mohn AR, Caron MG. Genetic animal models: focus on schizophrenia. Trends Neurosci. 2001;24:527-33.

47. Powell CM, Miyakawa T. Schizophrenia-relevant behavioral testing in rodent models: a uniquely human disorder? Biol Psychiatry. 2006;59:1198-207.

48. Marcotte ER, Pearson DM, Srivastava LK. Animal models of schizophrenia: a critical review. J Psychiatry Neurosci. 2001;26:395-410.

49. Morozov A, Muzzio IA, Bourtchouladze R, Van-Strien N, Lapidus K, Yin D, et al. Rap1 couples CAMP signaling to a distinct pool of p42/44MAPK regulating excitability, synaptic plasticity, learning, and memory. Neuron. 2003:39:309-25.

50. Kelly MP, Stein JM, Vecsey CG, Favilla C, Yang X, Bizily SF, et al. Developmental etiology for neuroanatomical and cognitive deficits in mice overexpressing Galphas, a G-protein subunit genetically linked to schizophrenia. Mol Psychiatry. 2009;14:398-415.

51. Srivastava DP, Jones KA, Woolfrey KM, Burgdorf J, Russell TA, Kalmbach A et al. Social, communication, and cortical structural impairments in Epac2deficient mice. J Neurosci. 2012;32:11864-78.

52. Shoji H, Takao K, Hattori S, Miyakawa T. Age-related changes in behavior in C57BL/6J mice from young adulthood to middle age. Mol Brain. 2016:9:11.

53. Takao K, Miyakawa T. Light/dark transition test for mice. J Vis Exp. 2006; https://doi.org/10.3791/104.

54. Komada M, Takao K, Miyakawa T. Elevated plus maze for mice. J Vis Exp. 2008; https://doi.org/10.3791/1088.

55. Nakao A, Miki T, Shoji H, Nishi M, Takeshima H, Miyakawa T, et al. Comprehensive behavioral analysis of voltage-gated calcium channel beta-anchoring and -regulatory protein knockout mice. Front Behav Neurosci. 2015:9:141.

56. Shoji H, Takao K, Hattori S, Miyakawa T. Contextual and cued fear conditioning test using a video analyzing system in mice. J Vis Exp. 2014; https://doi.org/10.3791/50871.

\section{Ready to submit your research? Choose BMC and benefit from:}

- fast, convenient online submission

- thorough peer review by experienced researchers in your field

- rapid publication on acceptance

- support for research data, including large and complex data types

- gold Open Access which fosters wider collaboration and increased citations

- maximum visibility for your research: over $100 \mathrm{M}$ website views per year

At BMC, research is always in progress.

Learn more biomedcentral.com/submissions 